\title{
Advances in Characterizing Recently-Identified Molecular Actions of Melatonin: Clinical Implications
}

\author{
Russel J. Reiter, Ramaswamy Sharma, Sergio A. Rosales-Corral, \\ Ana Coto-Montes, Jose Antonio Boga, and Jerry Vriend
}

\section{Introduction}

Some system-wide functions/aspects of melatonin were identified decades ago, e.g., regulation of seasonal reproduction [51, 200, 201], photoperiodic control of pineal melatonin synthesis [122, 227], sleep initiation [31, 117], antioxidant actions [67, 197], circadian rhythm modulation [208, 279], cancer inhibition [27, 86, 163], etc. These aspects of melatonin are generally well known by the scientists working in the field, although the detailed mechanisms of these actions, in most cases, require further clarification. These functions are not discussed in detail in the current review, but they may be mentioned when they are germane to the discussion. Also, the functions of melatonin in plants $[18,61,62,194]$ are not considered even though

R. J. Reiter $(\bowtie)$

Department of Cell Systems and Anatomy, UT Health San Antonio, San Antonio, TX, USA

Department of Molecular Genetics, University of Lodz, Lodz, Poland

e-mail: reiter@uthscsa.edu

R. Sharma

Department of Cell Systems and Anatomy, UT Health San Antonio, San Antonio, TX, USA

S. A. Rosales-Corral

Centro de Investigación Biomédica de Occidente, Instituto Mexicano del Seguro Social, Guadalajara, Mexico

A. Coto-Montes

Department of Morphology and Cell Biology, University of Oviedo, Oviedo, Spain

J. A. Boga

Servicio de Microbiologia, Hospital Universitario Central de Asturias, Oviedo, Spain

J. Vriend

Department of Human Anatomy and Cell Science, Max Rady College of Medicine,

University of Manitoba, Winnipeg, MB, Canada 


\section{SYSTEMIC ACTIONS $\quad$ CELLULAR ACTIONS}

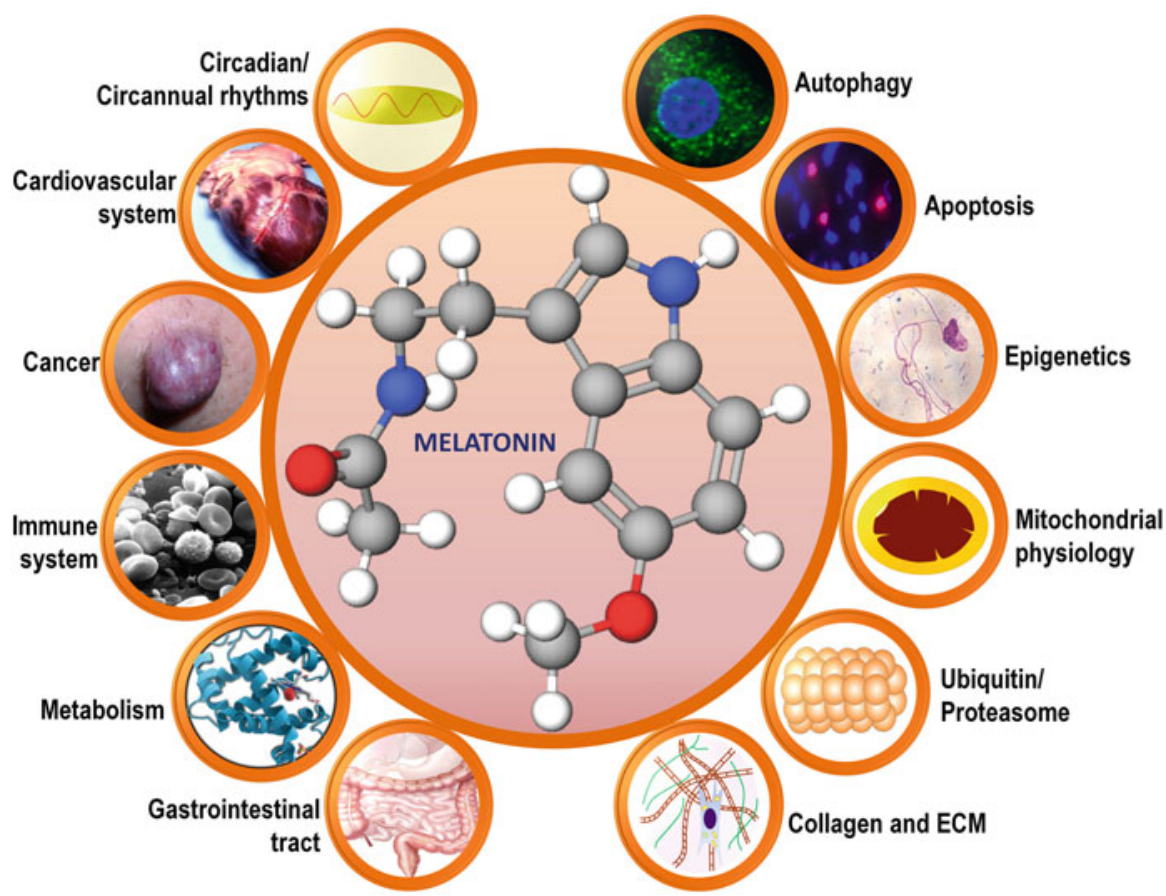

Fig. 1 Some of the identified systemic and cellular actions of endogenously-produced or exogenously-administered melatonin. There are other defined actions of melatonin that are not represented in this figure. The cellular actions illustrated are those considered in the current review. $\mathrm{ECM}=$ extracellular matrix

many of those actions have been widely investigated in recent years and these studies reveal that the actions of melatonin in plants are equally ubiquitous as its functions in animals.

What is briefly reviewed herein are some of the actions of melatonin at the cellular and subcellular levels that have implications for the more generalized processes mentioned (Fig. 1). Given the critical role of mitochondria in normal and pathological cellular function, the localization of melatonin in this organelle has broad implications for organ health and organismal well-being. For example, apoptosis and autophagy are important cellular processes that determine tissue architecture and organ physiology. Additional processes considered in this review are the role of melatonin in the regulation of epigenetics and of ubiquitin and proteasomal function. Finally, excessive deposits of collagen and extracellular matrix, accumulations collectively referred to as fibrosis, is a reflection of dysregulated cellular function which has severe negative consequences in a number of organs required for survival. This article by no means exhausts the large number of interactions melatonin has at the subcellular level. 


\section{Mitochondrial Physiology}

Mitochondria are double membrane-bound subcellular organelles which house the biochemical machinery for oxidative phosphorylation and energy, i.e., ATP, production. This pathway is located in the convoluted inner mitochondrial membrane whose molecular heritage includes the outer membrane of bacteria which, 2.5-1.5 billion years ago, were ingested by early eukaryotic cells in the process referred to as endosymbiosis. There are estimated to be thousands of mitochondria in every cell with cells that have a higher energy requirements, e.g., cardiomyocytes, being especially rich in this organelle.

An association of melatonin with mitochondrial physiology has been repeatedly confirmed within the last two decades although the molecular mechanisms by which melatonin accomplishes these tasks still require extensive investigation (Fig. 2). What was initially reported was that melatonin preserved the activities of mitochondrial complex I and IV in rats treated with the mitochondrial toxin, ruthenium red [153]; this toxin hinders the function of the mitochondrial $\mathrm{Ca}^{2+}$ uniporter which leads to the excessive production of damaging free radicals in these organelles. When toxin-treated animals were also given melatonin, but not when they were treated with either vitamin $\mathrm{E}$ or vitamin $\mathrm{C}$, the activities of the complexes were preserved; the toxin in this case was $t$-butyl hydroperoxide [154]. While the conserved mitochondrial function was consistent with melatonin's discovery as an antioxidant [187], the failure of vitamin C or E, molecules which also function as radical scavengers, is left unexplained. Perhaps the vitamins did not localize in sufficient amounts in the mitochondria to combat the large amounts of free radicals produced.

Findings reported by Martin et al. [153, 154] were the first that implied that melatonin may have a particular affinity for mitochondria, findings that were later verified (see below). The damage inflicted on the mitochondrial respiratory chain by toxin exposure was also reflected in the lowered ATP levels, an action also overcome by melatonin [155]. Shortly after the studies of Martin et al. [153-155], Okatani et al. $[173,174]$ also verified the beneficial actions on mitochondrial physiology by reporting that melatonin protects against hepatic mitochondrial injury due to ischemia-reperfusion and the deterioration that normally occurs in old mice.

Simultaneous with these studies, melatonin was reported to protect neural mitochondrial oxidative phosphorylation from other toxin exposures including 1-methyl4-phenylpyrimidine [1], 6-hydroxydopamine [49], and kainic acid [50]. These data further solidified the speculation that melatonin had high relevance to mitochondrial function.

The ability of melatonin to maintain mitochondrial respiratory chain function and ATP synthesis under conditions of hypoxia-reoxygenation and after mitochondriatargeted toxin exposure indicated that melatonin enters these organelles in sufficient amounts to counteract these caustic circumstances. That melatonin can quickly gain access to mitochondria was immunocytochemically verified by Jou et al. [109]. When rat brain astrocytes were incubated in medium containing the oxidizing 


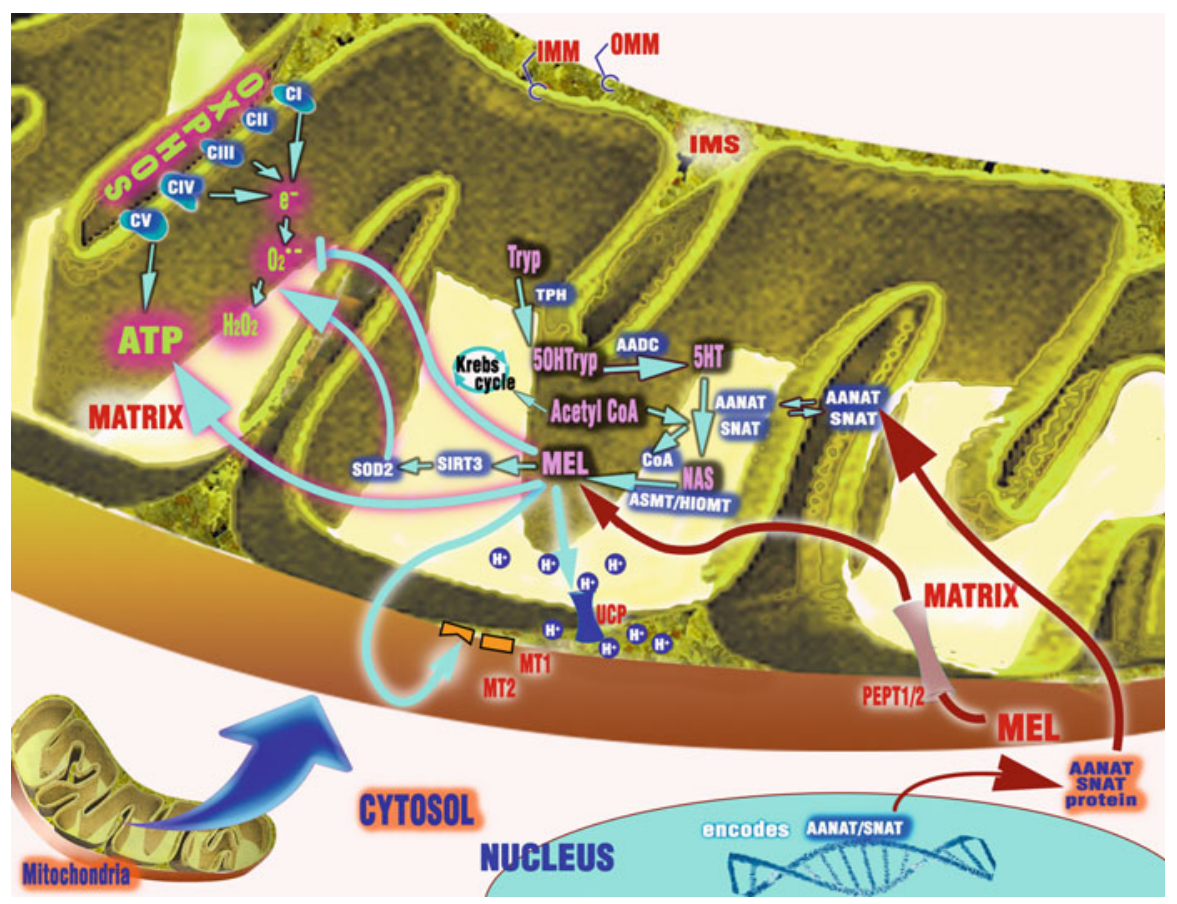

Fig. 2 This figure illustrates the facilitated transport of melatonin into mitochondria via the oligopeptide transporters, PEPT1 and PEPT2. The synthesis of melatonin in the mitochondrial matrix is also shown, a pathway that is aided by the ready availability of acetyl coenzyme A (acetyl $\mathrm{CoA}$ ); acetyl CoA is a co-factor for the activity of the acetyltransferase enzyme (SNAT/AANAT). Locally-produced melatonin may diffuse out of the mitochondria and interact with melatonin membrane receptors (MT1/MT2) on the outer mitochondrial membrane (OMM). By means of this action, melatonin may regulate cytochrome $\mathrm{c}$ release and subsequent caspase activation. Within mitochondria, melatonin scavenges reactive oxygen species and stimulates the antioxidative enzyme, superoxide dismutase 2 (SOD2), a pathway that involves SIRT3 and FOXO3a. Also, melatonin protects against the deterioration of oxidative phosphorylation (CI- CV) and enhances ATP production. By reducing mitochondrial oxidation dyshomeostasis, melatonin reduces the oxidation of cardiolipin and limits cytochrome $\mathrm{c}$ release. Melatonin also regulates the opening of the mitochondria transition pore directly and via an action on uncoupling protein (UCP). For many of these functions, additional data are required before these actions can be identified as being definitive. $\mathrm{IMM}=$ inner mitochondrial membrane; $\mathrm{IMS}=$ intermembrane space; OMM $=$ outer mitochondrial membrane; Try $=$ tryptophan; 5OHTry $=5$ - hydroxytryptophan; 5-HT = serotonin; NAS $=N$-acetylserotonin. Other details can be found in the text

agent, $\mathrm{H} 2 \mathrm{O} 2$, their mitochondria quickly exhibited intense fluorescence due to ROS generation. However, when mitochondria were incubated with $\mathrm{H} 2 \mathrm{O} 2$ and melatonin, the extreme mitochondrial fluorescent reaction that was associated with $\mathrm{H} 2 \mathrm{O} 2$ only was quenched. Like Martin et al. [154], Jou and co-workers [109] also found that vitamin E was significantly less effective than melatonin in reducing ROS-mediated mitochondrial fluorescence. The differences in the efficacies of melatonin versus vitamin $\mathrm{E}$ at the mitochondrial level were also apparent when the apoptotic indices of 
the astrocytes were compared; thus, melatonin was more efficacious in limiting mitochondrial-mediated apoptosis than was vitamin E. Jou et al. [110, 111] extended the evidence related to the functional preservation of mitochondria when they found that intracellular calcium dysregulation, mitochondrial transition pore opening, cardiolipin depletion and, cytochrome c release were attenuated in astrocytes challenged by oxidizing situations but not when treated with melatonin.

Biochemically, Maity et al. [149] and Zavodnik et al. [278] also reported that mitochondrial physiology of both gastric mucosal cells and hepatocytes, respectively, were maintained by treatment of animals with melatonin. In these studies, the mucosal cells were protected from the commonly-used drug, indomethacin, while the liver cells functioned normally in diabetic animals. Both of these toxicities are common in humans, so the findings have clear clinical relevance.

While it was well established that a number of tissues, in addition to the pineal gland, produce melatonin [4], the evidence remained circumstantial that the indoleamine was actually present in mitochondria. In 2012, Venegas et al. [256] were the first to estimate radioimmunoassayable levels in several compartments of fractionated neural and liver cells. Contrary to expectations, they observed very wide differences in the melatonin concentrations in different portions of the cells. In general, nuclear and cytosolic levels were low while mitochondrial measurements showed they were more than 10 times higher than in the other two compartments. This finding is strongly supportive of the involvement of melatonin with mitochondrial physiology.

Also of interest is that the concentrations of melatonin in different compartments did not vary over a 24-hour light:dark cycle (as do pineal and blood levels) and surgical removal of the pineal gland did not alter melatonin concentrations in any portion of the cells, except for the membrane fraction where melatonin levels actually increased. In addition to indicating the high likelihood of the importance of melatonin in mitochondrial physiology, it leaves in doubt, an answer to the question: what constitutes a physiological level of melatonin? [193]. The answer to this question is confounded because within subcellular organelles, melatonin levels vary widely and likewise, the concentrations of melatonin in different body fluids are greatly different.

The seemingly special association of melatonin with mitochondria $[3,195,196]$ prompted an examination of the evolutionary heritage of this widely-distributed indoleamine. In 1995, Manchester et al. [150] had reported that a bacterium (Rhodospirillum rubrum) contained immunoreactive melatonin. Considering this finding and the alleged origin of both chloroplasts and mitochondria from bacteria that were ingested as food by early eukaryotes (Endosymbiotic Theory), we [244] proposed that melatonin initially evolved in bacteria several billion years ago and when they were ingested and developed into mitochondria in eukaryotes, they retained their melatonin-forming ability.

This hypothesis has now been supported by the reports of He et al. [82] and Suofu et al. [233]. He et al. [82] showed that when isolated mouse oocyte mitochondria were incubated in medium containing the necessary precursor, serotonin, melatonin levels increased quickly in both the mitochondria and in the incubation medium. In 
the absence of available serotonin, no melatonin was formed. Meanwhile, Suofu et al. [233] approached the issue in a different manner by identifying the enzymes that synthesize melatonin from serotonin [35], i.e., $N$-acetyltransferase and acetylserotonin methyltransferase, in non-synaptosomal neural cell mitochondria. As with He et al. [82], when they incubated neurally-derived mitochondria with serotonin, these organelles formed melatonin and melatonin metabolites. Unlike melatonin production in the pineal gland, brain mitochondria did not exhibit a day: night rhythm in mitochondria consistent with the findings of Venegas et al. [256]. Based on the results of these two studies, it seems apparent that mitochondria have the necessary enzymes to synthesize their own melatonin.

Melatonin produced in these subcellular organelles is not released into the systemic circulation in any significant amounts; it, however, may be released from cells to mediate autocrine and paracrine actions. Additionally, mitochondriaproduced melatonin likely acts as an antioxidant in these structures, since they are major sites of free radical generation. The antioxidant functions could be achieved by direct radical scavenging [77] and/or by stimulating antioxidant enzymes [198].

In addition to its presumed local synthesis in mitochondria of all cells, exogenously- administered melatonin is also quickly extracted from the blood and distributed to mitochondria especially but also to other organelles (membranes, cytosol and nuclei) in cardiomyocytes [6]. The subcellular uptake was dose-dependent with the mitochondria and nuclei concentrating melatonin most rapidly after its peripheral administration; however, the melatonin concentrations in the mitochondria were about tenfold higher than those in the nuclei.

How melatonin gains access to cells and subcellular compartments has been extensively debated $[161,162]$. Several options have been considered. Being highly lipid soluble, melatonin could presumably simply passively diffuse through cell membranes. Alternatively, it was recently suggested that melatonin is actively transferred into cells through the glucose transporter, GLUT1 [84]. Huo et al. [101] proposed that the uptake of melatonin by cells and by mitochondria is a facilitated process that involves the oligopeptide transporters, PEPT1 and PEPT2. These transporters exist in both the membrane and mitochondria of the cells (human cancer cells) that were tested. Thus, at this point there are at least three options to explain the uptake and differential intracellular distribution of melatonin.

The high concentrations of melatonin in mitochondria is very fortuitous considering these organelles are major sources of damaging ROS and melatonin is a potent direct free radical scavenger [243] and indirect antioxidant (stimulation of antioxidant enzymes) [198, 205]. Even when compared with synthetically-modified antioxidants, i.e., Mito E and Mito Q, which concentrate in the mitochondria up to 500-fold over that of unaltered vitamin E or coenzyme Q10, at equimolar concentrations melatonin was still more effective in preserving cellular and mitochondrial physiology [144].

Melatonin obviously has numerous essential functions in mitochondria which are critical to the optimal functioning of these organelles (Fig. 2) and, therefore, cells/ organs as a whole. It is speculated that, as with pineal melatonin production, its synthesis in mitochondria of extrapineal tissues also wanes with age [210]. Several 
recent reviews summarize the absolute importance of optimally-preserved mitochondrial functions, all of which are maintained in some manner by melatonin $[5,79,180$, $188,268]$.

\section{Autophagy}

Autophagy is a self-degradative process which, under basal conditions, allows cells to remove misfolded proteins and damaged organelles. Starvation, growth factor deprivation, hypoxia, oxidation of critical molecules, protein aggregation, DNA damage or infection by intracellular pathogens are some of the conditions of cellular stress, which can induce autophagy.

This is a complex process including several sequential steps at molecular and cellular levels. The first step is the formation of a double membrane structure, the phagophore, which is generated de novo from plasma membrane-derived endocytic organelles such as endoplasmic reticulum or Golgi apparatus. This involves the mediation of ULK1/FIP200/ATG101/ATG13 protein kinase and VPS34/beclin1/ VPS15/ATG14 lipid kinase complexes. Both complexes induce the formation of the ATG5/ATG12/ATG16L1 complex (formed with the help of Atg7 and Atg10), which promotes the elongation of the phagophore engulfing a portion of the cytosol or specific cargoes (proteins, lipids, organelles), forming a double-membrane-bound vacuole called the autophagosome. Aided by Atg3 and Atg7, these complexes facilitate the addition of a phosphatidylethanolamine group to the cytosolic form of mammalian LC3 homologues (LC3A, LC3B, LC3C, Gabarap, Gabarap-L1, and Gabarap-L2), which is referred to as the LC3-I to LC3- II conversion. The lipidbound form of LC3 homologues is then recruited to the autophagosome, which fuses with a lysosome, forming a single-membrane-bound vesicle termed the autolysosome. Several lysosomal membrane proteins (GTPase RAB7, LAMP1, LAMP2, as well as SNARE proteins, such as syntaxin 17 and SNAP29) degraded the contents with the aid of another beclin1/VPS34 complex, where the UV radiation resistance-associated gene (UVRAG), rather than Atg 14, is required [129].

The basal level of autophagy is required to control protein conformation and maintenance of cell homeostasis and survival. Nevertheless, depending on the stress and cell types, autophagy plays a dual role either as pro-survival or as pro-death event. Autophagy participates in many cellular and physiological responses. Several studies have shown that some of these responses can be modulated by melatonin.

Autophagy plays an important role in initiation and progression of neurodegenerative diseases since they are characterized by the misfolding and aggregation of cellular proteins, which must be eliminated or they may become toxic. The protective role of melatonin in the regulation of autophagy has been reported in several of neurodegenerative diseases. Parkinson disease is characterized by the aggregation of $\alpha$-synuclein in dopaminergic neurons causing gradual degeneration of certain brain regions. The protective role of melatonin via autophagic processes in experimental models of Parkinson disease has been reported [2]. Thus, the exposure of glial cell 
type C6 or mouse striatal cells to 1-methyl-4- phenyl-1, 2, 3, 6-tetrahydropyridine (MPTP) provokes in increase of LC3-II, mediated by upregulation of the cyclindependent kinase 5 (CDK5), inducing $\alpha$-synuclein aggregation, which is reduced by pretreatment of mice with melatonin [230]. Rotenone, a Parkinson disease inducer, promoted autophagic cell death mediated by Bax and Omi release into the cytoplasm. A decrease in the expression of Bax and a drop in the release of Omi into the cytoplasm, as well as reduced cell death, was observed in Hela cells pre-treated with melatonin [288]. Another Parkinson disease inducer, kainic acid, increased $\alpha$-synuclein and the level of LC3-II and promoted neuronal loss in the hippocampus of mice. Melatonin enhanced $\alpha$-synuclein ubiquitination and reduced LC3-II, cathepsin B and lysosomal-associated membrane protein 2 (LAMP-2) [36].

Autophagy plays a crucial role in the neural damage induced by several neurotoxic agents, such as arsenite and cadmium. In these conditions, melatonin exhibited neuroprotective effects by regulating autophagy. Thus, melatonin inhibited arseniteinduced autophagy and autolysosome formation in rat primary cultured cortical neurons [247]. Also, the reduced autophagosome-lysosome fusion and inhibition of lysosomal function triggered by cadmium intake is reversed by treatment with melatonin in mouse neuroblastoma cells $[138,139]$. Oxaliplatin, which is a chemotherapeutic agent widely used in the treatment of different cancers, induces neuropathy as a secondary effect. Oxaliplatin-treated rats showed impaired autophagy with basal levels of LC3A/3B-I and II, beclin, Atg3, Atg5, and Atg7 being attenuated in the sciatic nerve and dorsal root ganglia. An increase of these autophagic proteins was observed in melatonin-treated rats [17].

Glioblastoma multiforme is an aggressive tumor with a high mortality rate. Melatonin elevated LC3-II and induced progressive accumulation of autophagosome vacuoles via Akt activation in treated glioblastoma-initiating cells (GICs) [156].

While a basal autophagy rate has a protective effect during heart failure, ischemic cardiomyopathy, and cardiac hypertrophy, excessive autophagy promotes cardiac atrophy [176]. Diabetic cardiomyopathy is considered a major cause of heart failure. In melatonin-treated diabetic animals, adverse left ventricle remodeling was alleviated and cardiac dysfunction was reduced by enhanced Sirt1 expression and reduction of Mst1, which have pivotal roles in autophagy induction [281-283]. Chronic intermittent hypoxia (CIH), which occurs during obstructive sleep apnea syndrome (OSAS), causes multiple cardiovascular disorders such as coronary heart disease, hypertension and myocardial hypertrophy [72]. A higher LC3II/I ratio and greater Beclin1 expression in myocardial tissue of rats with $\mathrm{CIH}$-induced myocardial hypertrophy suggest an increased autophagic response. Administration of melatonin induced additional autophagy via activation of AMPK, thereby having a protective effect in CIH rats [269]. Doxorubicin (DXR), which is an important chemotherapeutic agent, causes cardiotoxicity as a major side effect. Increased autophagy, which is upregulated during DXR-induced cardiotoxicity, is concomitant with a lower cell death [224]. The deteriorative effects of DXR on mitochondria are reduced by melatonin in an experimental model of cardiorenal syndrome; in this situation, melatonin reversed the drop in ATP production and inhibited cytochrome $\mathrm{c}$ 
release from mitochondria. It appears that melatonin has significant protective effect by modulating mitophagy, a process that removes damaged mitochondria through autophagy [42].

The potential benefit of melatonin on the gastrointestinal system due to the regulation of autophagy has been examined. The liver, which is the main organ for detoxification of hazard agents, is often dysregulated by toxic agents such as cadmium. Mitochondrial loss, cellular energy mitigation and cell death are a consequence of cadmium-induced hepatotoxicity resulting from excessive autophagy. Melatonin reduced mitochondrial reactive oxygen species (ROS) and subsequently lowered autophagy and cell death in HepG2 cells by activation of SIRT3-SOD2 signaling [186].

Carbon tetrachloride (CCL4) has been used to induce experimental hepatic fibrosis, which is overly exuberant wound healing in which excessive connective tissue accumulates in the liver. The rise in beclin1, Atg12, Atg5, and Atg16L1 mRNA levels observed in CCL4- induced hepatic fibrosis are reversed in melatonin-treated mice [212].

A diet rich in energy together with a sedentary life style contributes to obesity, which has become a common problem in developed countries. Fatty liver disease in a range of conditions caused by a build-up of fat in the liver, frequently found in obese subjects. The role of melatonin as a regulator of autophagy in obesity has been studied in the liver of obese, leptin-deficient mutant mice (ob/ob mice). One report claimed these animals had downregulated autophagy, which was enhanced by melatonin treatment associated with a decline in beclin1 and a rise in p62 [52].

Several studies have documented a role of melatonin in several gastrointestinal tumors. Thus, in HepG2 cells, which were derived from a male in a hepatocellular carcinoma, melatonin induced autophagy through activation of JNK but not mTOR [175]. In human colorectal cancer cells (HCT116 cells), melatonin treatment activated both autophagy and apoptosis by upregulation of both pro-apoptotic Bax and Bcl-xL [93]. Colorectal cancer (CRC) that develops in patients in inflammatory bowel disease is known as colitis- associated colorectal cancer (CAC); a model of this cancer type in mice uses 1, 2- dimethylhydrazine dihydrochloride administration. In this animal model, melatonin reduced CAC-induced autophagy as revealed by the expression pattern of various autophagy markers such as beclin1, LC3B-II/ LC3B-I ratio and p62 [252]. Melatonin also induces the autophagic pathway in tongue squamous cell carcinoma cell line, Cal 27, as evidenced by the upregulation of LC3-II and downregulation of SQSTM1/P62 [61]. Valproic acid (VA) inhibits invasiveness of bladder cancer. When combined with melatonin, VA exhibits enhanced autophagy by triggering several autophagic genes including Beclin1, Atg3 and Atg5 [143]. Collectively, the results support the use of melatonin as a chemotherapeutic in the treatment of these tumors of the gastrointestinal system due to their ability to enhance cancer cell autophagy.

Melatonin plays various modulatory roles in cellular physiology. For example, autophagy is necessary for the preservation of normal morphology, cell mass, and function of pancreatic $\beta$ cells. AR42J cells, derived initially from a transplantable tumor of a rat exocrine pancreas and used as a model of acute pancreatitis, showed an 
increased autophagy via endoplasmic reticulum stress. Melatonin enhanced autophagy in this experimental model [38].

Human fetal osteoblastic (hFOB1.19) cells are used as model of osteoporosis. An increase in glucose in these cells promoted autophagy, which was reduced by melatonin through inhibition of the ERK pathway.

The Harderian gland cells of the Syrian hamster are exposed to elevated oxidative stress because of their high content of porphyrins. To maintain the function of these glands, many of these cells exhibit autophagic processes. In these cells, melatonin reduced the destructive effects of free radicals via different mechanisms including amelioration of detachment-induced autophagic cell death [255].

Melatonin has beneficial effects on the maturation of oocytes by induction of autophagy and enhancing the expression of a number of genes including ATG7 and beclin1, as seen in pig oocytes and cumulus cells [39].

Autophagy can also be induced during different stages of an infection. Although autophagy can limit the cytopathic effect of pathogens and the pathological consequences via a cellular process referred to as xenophagy, some cells have developed strategies to directly or indirectly subvert autophagy in order to promote different stages of the cell cycle.

Vibrio vulnificus hemolysin (VvhA) induces apoptosis and autophagy in human intestinal epithelial (HCT116) cells. Melatonin inhibited JNK-mediated phosphorylation of Bcl-2 responsible for the release of Beclin1 and Atg 5 expression, thereby blocking VvhA -mediated apoptotic and autophagic cell death [134].

Rabbit hemorrhagic disease virus (RHDV) and rabbit vesivirus ( $\mathrm{RaV})$, two members of the genus Lagovirus (Family Caliciviridae), cause autophagosome and autophagolysosome formation [211]. During RHDV-induced autophagy, increased expression of beclin1, LC3-II/CL-I ration and Atg5-Atg12-Atg16L1 was found. A dysfunctional autophagy with impairment of the autophagic flux was also detected, a judgment based on a parallel rise in p62/SQSTM1 expression. A reduction of the level of these autophagic proteins by melatonin treatment indicates a drop in the autophagic response. Melatonin administration triggers similar mechanisms in other RNA viruses, such as HCV, whose infection cause a similar dysfunctional autophagy [211].

Prion proteins induce misfolding of normal cellular proteins and they are causative of several neurodegenerative diseases, such as Kuru and Creutzfeldt-Jacob disease. Melatonin protects SH-SY5Y cells from PrP-induced neurotoxicity by enhancing LC3-II levels and inducing autophagy [108].

Autophagy has a crucial role in homeostasis and in human diseases since it causes cellular survival or death depending on the severity of the damage. Several studies have reported that melatonin either promotes or suppresses autophagy, which implies that melatonin can balance the autophagic process. It is clearly established that melatonin's actions are context specific; these differential actions explain its beneficial effects and support the use of this molecule as a potential therapy for management of certain diseases (Fig. 3) where autophagy plays an important role. This brief summary elaborates only at the few sites/conditions in which melatonin has been shown to influence autophagic processes. 


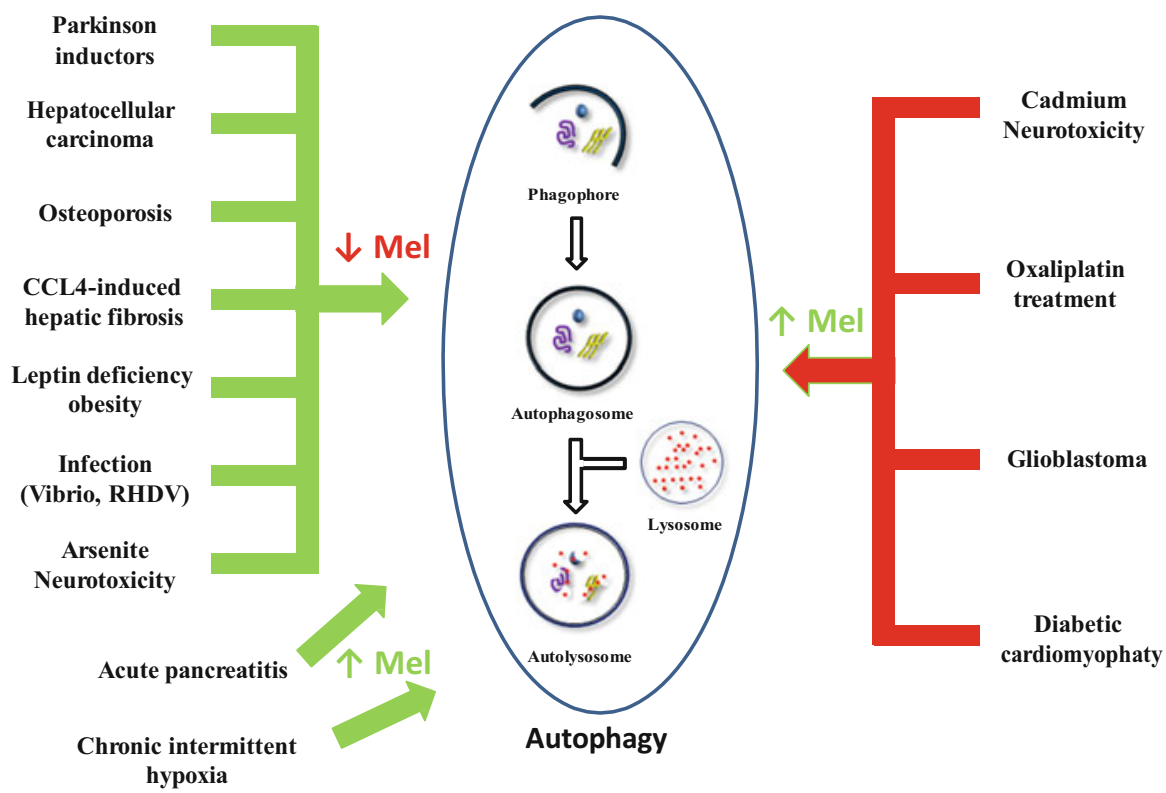

Fig. 3 Autophagy is induced (green rows) or inhibited (red rows) in several diseases, clinical conditions and experimental models. Melatonin administration counteracts or enhances such induction or inhibition

\section{Apoptosis}

Cell death is a necessary part of the normal development and maturation cycle. This process is the result of different cellular mechanisms that the cell initiates in response to both physiological and pathological stimuli. The cellular mechanisms are mainly encompassed by two essential processes: apoptosis and autophagy, although the special conditions of each cell can give rise to intermediate and mixed processes that can unleash cell death with characteristics common to both. Even a sustained and massive loss of energy may lead to cell death, such as necrosis.

Melatonin is produced rhythmically in the pineal gland and arrhythmically in the mitochondria of every cell. Via its membrane receptors, MT1 and MT2 [58, 142], or after it enters cells, melatonin modifies cell processes in a variety of ways to mediate cell death. These modifications, although they are usually intimately related to oxidative stress, are dependent on cell and tissue type and on the specific conditions in which they are found. Detailed studies have clarified some of the mechanisms involved in apoptosis as well as the similarities and differences that exist between the variations in programmed cell death.

Apoptosis of a cell induces a series of changes including cell shrinkage, blebbing of the plasma membrane, maintenance of organelle integrity, condensation and fragmentation of DNA, and eventually, ordered removal of the cell by phagocytes [118]. The triggering processes for apoptosis, extrinsic or receptor-mediated and 
intrinsic or mitochondria-mediated pathways, are complex and are partially known [185]. Recent evidence shows non- apoptotic roles for many usual effectors of the apoptotic signaling pathways. Thus, caspase 2, one of the best known molecules related to the triggering of apoptosis, is also involved in cell cycle regulation and DNA repair [254]. These new discoveries have complicated the understanding of the regulation of apoptosis.

Apoptotic events are highly regulated by the Bcl-2 family of proteins, which shares homology among the four common Bcl-2 domains. This family includes antiapoptotic proteins including $\mathrm{Bcl}-2, \mathrm{Bcl}-\mathrm{XL}$ and $\mathrm{Mcl}-1$, that possess all the four (BH) domains and act on mitochondria [29], endoplasmic reticulum and/or the nucleus [45]. The relationship that these proteins establish with free radicals has been identified and they have common actions on mitochondria [37]. Pro-apoptotic proteins are also included in Bcl-2 family. These proteins contain BH domains 1, 2, 3 , unlike the anti- apoptotic proteins. The pro-apoptotic proteins include Bax and Bak which are related to the BH3-only proteins, i.e., Bim, Bad, Bmf, Noxa and Puma [95]. They function primarily to neutralize the anti-apoptotic proteins by sequestration and creation of heterodimers [103].

The influence of reactive oxygen species (ROS) on cell death signaling is twofold. Exposure of cells to a pro-oxidant state induces oxidation of caspases that prevent cells from undergoing apoptosis [44, 184]. Elevated intracellular ROS, e.g., $\mathrm{H} 2 \mathrm{O} 2$, levels cause cytoplasmic acidification that influences the conformational status of proteins of the Bcl-2 family, resulting in their activation and facilitating the release of apoptosis amplification factors from the mitochondrial intermembrane space [7, 87]. This dual effect of ROS on apoptosis suggests an explanation of the actions of melatonin on the programmed cell death type since a major function of melatonin is as an antioxidant. However, melatonin's pleiotropic actions also make it difficult to identify the specific mechanisms by which melatonin acts on apoptosis. Herein, some of the multiple processes by which melatonin modulates apoptosis are considered.

Melatonin has multiple means by which it modifies and/or regulates apoptosis in both cancer and in normal cells. Melatonin's actions in these cell types are opposite to one another; it is pro-apoptotic in cancer cells and anti-apoptotic in normal cells. The mechanisms of these differential actions are still to be clarified.

Although melatonin typically exerts beneficial effects on cells, its action, via its receptors, due to its antioxidant properties or by alternative mechanisms, may vary depending on the tissue studied and on the melatonin dose used. Thus, melatonin alleviates liver damage by reducing mitochondrial dysfunction resulting from oxidative damage which is hyperglycemia- induced [125, 126]. Similarly, melatonin reduces oxidative stress and hepatic apoptosis promoted by ethanol administration $[165,177]$. In both cases, scavenging of ROS may be the major explanation for its anti-apoptotic effects. Moreover, melatonin is also efficient in the prevention of apoptosis by acting through its MT2 membrane receptor [218] in bile duct-ligated young rats or inhibiting endoplasmic reticulum stress, a process that often induces apoptosis [53] in leptin-deficient mice. Similar mechanisms of protection are shown 
when melatonin reverses bone loss due to its antioxidant actions that prevent antiapoptotic events [147].

Osteoporosis and several other diseases increase in prevalence with age, simultaneous with the age-related drop in peak nocturnal melatonin secretion. Several articles have confirmed that this coincidence is not accidental, with the loss of melatonin being related to aggravation or an increase in the incidence of the ailment $[13,169]$. Thus, during aging, the drop in the nocturnal melatonin peak is associated with a rise in bone resorption, suggesting that melatonin may act as an endogenous osteoclast inhibitor [178]. Melatonin has been shown to limit bone resorption by limiting osteoclastogenesis $[88,127]$. Since the osteoclastic actions involve apoptosis, melatonin indirectly inhibits cell death [152].

Heart failure is also a multifactorial syndrome that increases in prevalence with age [169]. Heart failure involves cardiomyocyte apoptosis; this is a major physiopathological feature and melatonin reverses it [56, 167, 179, 281-283]. In mouse models of post-infarction damage, melatonin reduces apoptosis through Mst1/Sirt1 signaling [98]. In a diabetic model, melatonin lowers apoptosis by modulating the related proteins [11]. Zhang and coworkers, in the same model, also observed a drop in apoptosis by after melatonin administration, while also conserving mitochondrial integrity and biogenesis [281-283]. The improved mitochondrial efficiency would have a direct impact on the inhibition of the intrinsic pathway of apoptosis.

Another means has been recently described by which melatonin modulates, and finally inhibits, apoptosis [114]. This mechanism involves neither membrane receptors (MT1, MT2) nor melatonin's antioxidant effect. This action involves the ubiquitination of target proteins in the apoptosis pathway, which is a usual mechanism that reduces Bcl-2 proteins and regulates apoptosis [264]. In porcine granulosa cells, melatonin limits BimEL, ubiquitinating it so it can be degraded by the proteasome [114]. This latter action not only identifies a new action of melatonin, but also may help to explain the differential behavior of melatonin in normal cells and cancer cells. In cancer cells, melatonin has been described as a potent proteasome inhibitor [258, 259]. Although many of the studies that show an inhibitory role of melatonin on apoptosis in normal cells may lack detailed mechanisms, the anti-apoptotic effect of melatonin deserves to be studied in greater detail considering the significant therapeutic repercussions that its use would imply.

The brain is highly sensitive to oxidative stress because it consumes a large amount of oxygen and generates more ROS than most other tissues. Moreover, it is rich in easily-oxidizable polyunsaturated fatty acids and endowed with relatively low levels of endogenous antioxidants [147, 191]. This makes it a clear target in which to examine the role of antioxidants on apoptosis. Melatonin has been shown in multiple studies to be a highly effective neuroprotective agent not only in neurodegenerative diseases [206, 266, 281-283], but also after brain injury [147]. These protective actions are related to the functions of melatonin as an antioxidant and antiapoptotic actions. In mice, melatonin may protect against ischemic stroke via an action on MT2 receptors [40]. During brain ischemia-reperfusion, melatonin reduces upregulation of Nox 2 and Nox4 expression, enhancing its anti-apoptotic capacity 
[137]. Anti-apoptotic actions of melatonin, mediated by membrane receptors, are not confined to brain damage but also have been observed under neurobehavioral dysfunctions [265, 280].

Melatonin also reduces deleterious apoptotic processes in the central nervous system because of its ability to limit the activity of calpains either directly or by increasing the activity of calpastatin, a major inhibitor of calpains [241]. After spinal cord injury, an increased concentration of $\mathrm{Ca}^{2+}$ induces an activation of the calpains. Caspase- 3 together with the calpains, causes the death of the neurons by apoptosis [15]. Melatonin administration reduces calpain gene expression, caspase-3 activity and, thereby, neuronal death in animals with spinal cord injury [209]. Methamphetamine is a neurotoxic molecule that causes neuronal apoptosis and activates glial cells in the central nervous system. It functions to reduce cell viability by depleting calpastatin levels which upregulate calpains and caspases [241]. Melatonin reverses the depletion of calpastatin and improves mitochondrial dysfunction thereby lowering cell death by reducing apoptosis [234, 235]. A similar effect of melatonin is observed during dexamethasone- induced neurotoxicity [236].

Stem cells are undifferentiated cells that can transform into multiple cell types. Stem cells are classified into several categories: totipotent stem cells which can give rise to any cell type; pluripotent stem cells, which differentiate into many tissue cell types, but not into a functional organism, and multipotent stem cells which can differentiate into a limited number of tissues. Stem cells have a huge therapeutic potential in a variety of diseases and they become essential to repair and regenerate damaged tissues or organs. However, the low survival rate of engrafted stem cells remains an important obstacle for stem cell therapy. It is essential to identify molecules capable of reducing cell death of stem cells and recent advances have indicated melatonin may be one such molecule.

Due to the antioxidant action of melatonin, it readily controls oxidative stress in stem cells [281-283] as in differentiated cells as described above. Apoptosis induced by oxidative stress is negated by melatonin since it directly neutralizes ROS [253] or indirectly removes them by the activation of antioxidant enzymes [65]. In the case of stem cells, it is noted that melatonin does not block the differentiation capacity of these cells by reducing free radicals; this is important since the increase in free radicals is a usual signal that leads to differentiation. Some authors, however, emphasize that "melatonin might abolish redundant free radicals" [65]. In fact, melatonin is not a differentiation- blocking agent, but is, instead, able to promote differentiation as several authors have noted [181, 245].

The role of melatonin as anti-apoptotic protector in toti- or pluripotent stem cells is tissue-dependent; as a consequence, caution should be exercised in extrapolating molecular mechanisms discovered in one cell type to other stem cells. In human hexokinase 2 (HK2) cells, an immortalized proximal tubule epithelial cell line, melatonin counteracts the deleterious effects of cisplatin, inducing its anti-apoptotic actions [281-283]. Only a few reports have identified the intermediate events in this process. ERK, through a melatonin receptor- independent process, seems to be 


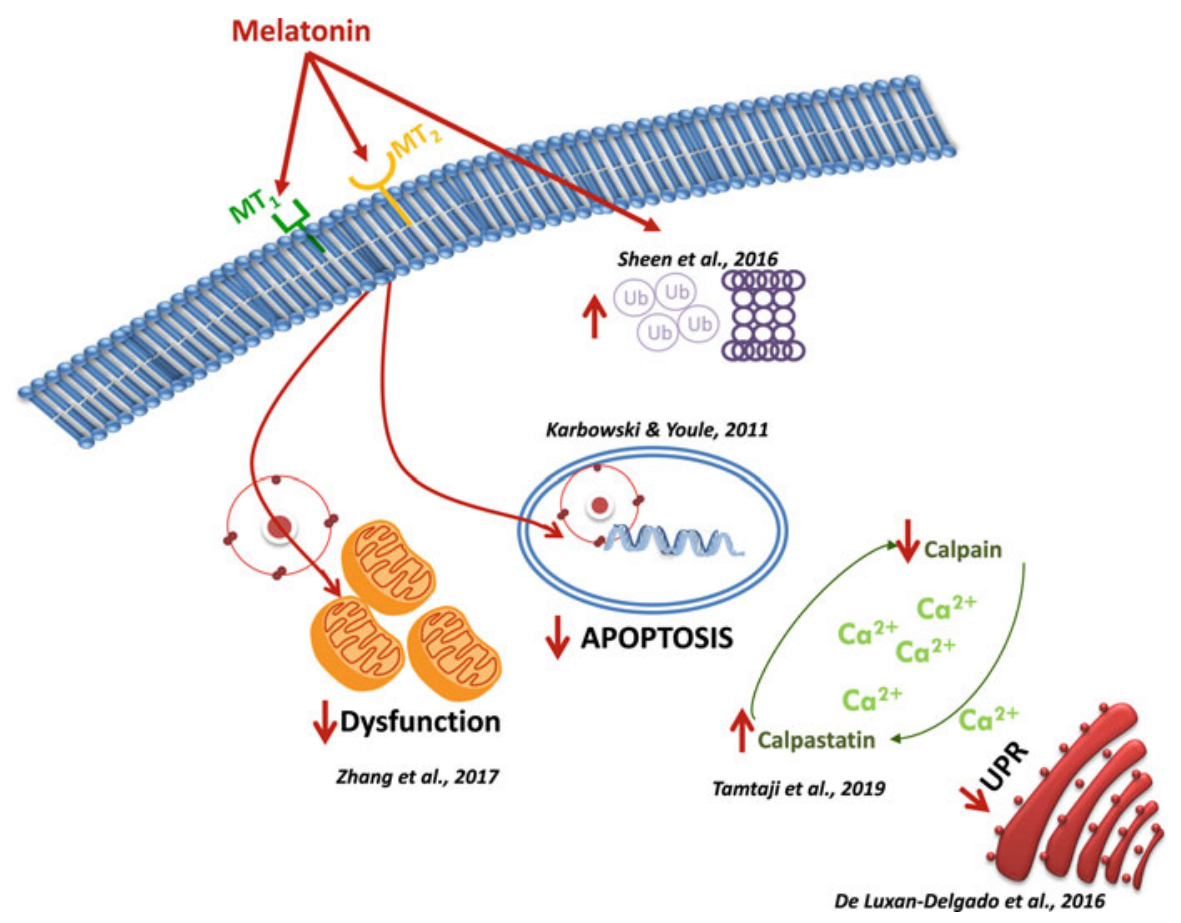

Fig. 4 This figure depicts some of the targets of melatonin to prevent apoptosis in normal cells. Three major action pathways have been described: through its membrane receptors MT1 and MT2, as a direct antioxidant and/or promoting ubiquitination. The first two act by performance optimization of essential organelles such as the endoplasmic reticulum and the unfolded protein response (UPR) and improving the mitochondrial physiology. The third one leads to protein destruction through the proteasome. In a potential minor pathway, melatonin directly acts on molecules involved in apoptosis, such as calpains and calpastatins that regulate apoptosis due to its action on caspases, or on proteins located in the mitochondria, favoring an increase of mitochondrial efficiency and reducing free radicals production

involved [145, 249]. Also, the regulation of expression BAX/Bcl-2 ratio and disruption of mitochondrial membrane potential have been observed [266]. MT1 and MT2 melatonin membrane-receptor may not be related to these anti-apoptotic effects of melatonin in different types of stem cells. Clearly, additional detailed reports related to the cellular mechanisms by which melatonin modulates apoptosis during stem cell-based therapy are required.

Considering the ubiquitous and essential nature of programmed cell death, it has implications to many normal developmental processes and is surely important in a variety of pathophysiological conditions. Many of these actions are summarized in Fig. 4 with an indication of their clinical relevance. 


\section{Ubiquitin-Proteasome System}

We recently suggested a likely relationship between melatonin, ubiquitin and the proteasome [258, 259]. A fairly well documented connection between melatonin and the ubiquitin-proteasome system (UPS) in the modulation of the synthesis and degradation of the rate limiting enzyme involved in melatonin production, AANAT (arylalkylamine $\mathrm{N}$ - acetyltransferase), by ubiquitin ligases in the pineal gland has been documented [120]. The regulation of AANAT synthesis in the pineal gland by its adrenergic innervation and the cAMP/PKA pathway has been described in detail $[89,120,121]$. These investigations reported the rapid decrease in AANAT protein induced by L-propranolol was blocked by proteasome inhibitors [68]. They concluded that the rapid drop in AANAT protein and AANAT activity induced by light exposure or propranolol administration was due to proteasomal degradation of AANAT. Additional data documenting a rise in AANAT in the pineal gland after administration of proteasome inhibitors was reported by Huang et al. [100]. These investigators also pointed out that stabilization of AANAT protein was regulated by phosphorylation and by interaction with a 14-3-3 protein.

The role of proteasome inhibitors on the synthesis of AANAT was also investigated. Ho et al. reported two different effects of proteasome inhibitors, an increase in norepinephrine (NE) stimulated AANAT protein in rat pinealocytes, but a reduction in AANAT transcription if the inhibitor was administered prior to stimulation with NE [90, 248]. Schomerus et al. [213] observed that, in bovine pinealocytes, there was little variation in AANAT transcription and proteasomal destruction of AANAT was more important than transcriptional regulation of AANAT.

The above-mentioned studies did not identify specific ubiquitin ligases or specific deubiquitinases regulating the transcription of AANAT or those modulating its destruction by the proteasome. Based on studies of the ubiquitin ligase Praja2, Lignitto et al. [140] found that Praja2 interacts directly with the regulatory component of PKA. Based on this data, Vriend et al. [263] included Praja2 in a model of AANAT control in the pineal gland. Considering the complexity of control mechanisms of AANAT, it is reasonable to speculate that more than one ubiquitin ligase is involved in regulation of AANAT degradation and transcription. Since CREB is a transcription factor involved in the transcription of AANAT [223, 257] the ubiquitin ligase CREBBP (CREB binding protein) would be a reasonable protein to investigate for its potential in regulating AANAT transcription. It was first isolated as a protein that binds to the transcription factor CREB (cAMP response element binding protein) [41]. The p65 component of NF-kB is reported to bind to CREBBP (aka CBP) [271].

The number of similarities between the actions of proteasome inhibitors and the actions of melatonin have been noted [258, 259]. Both are reported to have antiinflammatory activity mediated by inhibition of NF-kB activation and DNA bindingactivity [96, 99]; both upregulate antioxidant enzymes under conditions of oxidative stress [275] and stimulate transcription of genes of the NRF2-antioxidant response pathway [107, 260, 261]; both are reported as pro- apoptotic in cancer cells 
[70, 207]; both inhibit HIF-1 and VEGF [262, 267]; and, both interfere with cell cycle regulation [102, 221]. While it cannot be assumed that melatonin and proteasome inhibitors are equally effective, the similarities provide an interesting model for investigating the 'pleiotropic' effects of melatonin administration [221]. One explanation for the similarities of the actions of melatonin to that of proteasome inhibitors include the possibility that melatonin itself acts as a proteasome inhibitor or that it indirectly influences the UPS via an effect on phosphorylation of key proteins of the UPS [258, 259]. Other explanations may arise as research in this area develops.

Two major factors regulating the activity of proteins involved in these processes are phosphorylation and ubiquitination. Both, for example, are required for processing of proteins for degradation by the proteasome. Such is the case for proteins regulating NF-kB activation.

NF-kB is a transcription factor well studied for its role in induction of proteins of the immune response and inflammatory factors [281-283]. The NF-kB complex is formed by various combinations of the five Rel family members, p50, p52, p65 (Rel A), Rel B, and Rel C [158]. The most common dimer is p50/p65 (p50/RelA) and the term NF-kB is often used as a term equivalent to p50/RelA dimer. In the canonical NF-kB pathway the p50/RelA acts as a transcription factor after its translocation to the nucleus. In an alternative non-canonical pathway, the p52/RelB dimer acts as a transcription factor [92].

Proteins of the IkB family are important regulators of NF-kB, the best known being IkB $\alpha$. Cytoplasmic IkB $\alpha$ can rapidly prevent the translocation of p50/RelA to the nucleus [115], and reportedly can enter the nucleus to retrieve NF-kB [16], preventing NF-kB from interacting with DNA as a transcription factor. Phosphorylation and ubiquitination, however, make IkB $\alpha$ susceptible to proteasomal degradation, thereby activating NF-kB. Karin and Ben-Neriah [115] noted the serine/ threonine kinase, IKK, that phosphorylates IkB $\alpha$ as the 'key to NF-kB activation'. They concluded that IKK subunit, IKK $\beta$, is required for activation of NF-kB.

At least two ubiquitin ligases are important in regulating the NF-kB pathway. A specific ubiquitin ligase that binds to the $\mathrm{Nf}-\mathrm{KB} / \mathrm{IkB} \alpha$ complex was identified in 1998 by Yaron et al. [273]. It was named for its activity as an E3 ubiquitin ligase and for its binding to phosphorylated $\operatorname{IkB} \alpha, \mathrm{E} 3$ receptor subunit of $\operatorname{IkB} \alpha(\mathrm{E} 3 \mathrm{RSIkB} \alpha)$. It is now better known as the ubiquitin ligase $\beta$ - $\operatorname{TrCP}$, a ubiquitin ligase with several additional substrates. The substrates of $\beta$-TrCP include $\operatorname{IkB} \alpha, \operatorname{IkB} \beta, \operatorname{IkB} \varepsilon$ [81], p52 [12] and p105, the precursor of p50 [24]. Proteolytic processing of p105, to produce $\mathrm{p} 50$, is also proteasome-dependent [91]. The NF-kB subunit RelA is likewise subject to proteasome-dependent degradation [91].

A second ubiquitin ligase complex regulates the degradation of IKK. This ubiquitin complex [119, 132], Keapl-Cul3-Rbx, is better known for its role in the response to oxidative stress by regulation of NRF2 [272] and the antioxidant response element pathway.

Thus, activation of NF-kB, and its subsequent binding to DNA, requires the subtle dance of phosphorylation and ubiquitination of IkB and IKK. The precise role of melatonin in this dance has not been completely determined. There are numerous 
technically convincing reports that melatonin inhibits NF-kB activation and inhibits DNA binding of p65 under various experimental conditions [25, 43, 99, 219]. The reports of melatonin inhibition of NFkB activation and inhibition of DNA binding refer to the 50p/p65 (p50/RelA) form of NFkB [136, 189].

Li et al (2009) interpreted the melatonin-induced inhibition of NFkB binding to DNA to be the result of melatonin blocking the degradation of $\operatorname{IkB} \alpha$. Further data was obtained by two separate research groups [10, 219]. Shi et al. [219] reported that melatonin inhibited the formation of the phosphorylated form of $\mathrm{IkB} \alpha$, but not the non- phosphorylated from of $\mathrm{IkB} \alpha$ in a cell line stimulated with lipopolysaccharide (LPS). In a study of exercise in rats on NF-kB, Alonso et al noted that phosphorylated IkB $\alpha$ and IKK in muscle cells increased with exercise; the increase in both was prevented by melatonin [10]. Their data suggested that melatonin inhibited degradation of phosphorylated IkB $\alpha$ indirectly by inhibiting the activity of IKK. If IKK is the key to NF-kB activation, as suggested by Karin and Ben-Neriah [115], it could also be the key to understanding the mechanisms of melatonin in NF-kB activation. Since IKK $\beta$ is a substrate of the ubiquitin ligase Keap1 complex and is responsible for its ubiquitination [132, 250, 251], we suggest that the interaction of Keap1 and IKK may contribute to the mechanism by which melatonin administration activates NF-kB.

Much has been written on melatonin as an antioxidant [151, 197, 242]. One aspect of this is that melatonin stimulates the activity of antioxidant enzymes, including superoxide dismutase, glutathione peroxidase, heme oxygenase 1, and NADPH:quinoneoxidoreductase [65, 198, 205]. As noted, proteasome inhibitors also stimulate the activity of these enzymes. The components of the antioxidant stress response have been well described. They include the Keap1 ubiquitin ligase complex and the NRF2-antioxidant response element pathway. We reviewed the evidence that the antioxidant action of melatonin involves the Keap1-NRF2-ARE pathway in 2015 [260, 261]. We speculated that one possible mechanism that would explain the stimulation of antioxidant enzymes by melatonin was inhibition of the proteasome. While there was some evidence for this in vitro this has not been shown to be a direct effect [182].

Oxidative stress itself can impair the functional activity of the $26 \mathrm{~S}$ proteasome [8]. The smaller $20 \mathrm{~S}$ proteasome is less susceptible to such stress. It has been shown that the $20 \mathrm{~S}$ proteasome can degrade oxidized proteins, including SOD, independently of ubiquitin [222]. Thus, while melatonin very likely interacts with the KEAP2-NRF2-ARE pathway in simulating the production of antioxidant enzymes, its precise mechanism of action has not yet been determined.

Since melatonin influences a number of proteins associated with circadian rhythms [32], it is reasonable to investigate its possible role in regulation of clock genes. Gatfield and Schibler [69] provided evidence that cyclic expression of Cryl clock gene was controlled by the ubiquitin ligase FBXL3. Further evidence that cycles of ubiquitination and deubiquitination of clock genes contributed to circadian rhythms was reviewed by Stojkovic et al. [229]. Data supporting a role for melatonin in regulating expression of clock genes in the hypothalamus via the UPS was summarized in a review by Vriend and Reiter [260, 261]. 
The interaction of melatonin and mitochondria was reviewed in a series of manuscripts in the journal, Cellular and Molecular Life Sciences, in 2017. These reviews documented melatonin transport into the mitochondria [161, 162], a role for mitochondria in the antioxidant effect of melatonin [198-201], melatonin regulation of the electron transport chain [79], and melatonin interaction with glutathione redox cycling [34]; a direct action of melatonin on mitochondrial DNA has been proposed [188]. Melatonin is also reported to enhance mitochondrial biogenesis in rats with liver fibrosis [113] and the hepatic cells in culture [73].

The ubiquitin proteasome system could coordinate the various actions of melatonin associated with the mitochondria, but there is little information available on the topic. Lavie and co-authors estimated that 203 mitochondrial proteins were ubiquitinated [130] including outer and inner membrane proteins. They noted that the UPS promotes succinate dehydrogenase dependent oxygen consumption and increases ATP, malate and citrate levels and concluded that the UPS regulates energy metabolism in the mitochondria. The UPS also controls biogenesis of mitochondria [28] and mitophagy [30].

The ubiquitin proteasome system also plays a role in melatonin-induced autophagy. As noted above, Atg7 is one of the autophagic proteins influenced by melatonin administration. Atg7 is a ubiquitin activating enzyme (an E1 enzyme) [270]. This enzyme is found in species as diverse as Saccharmomyces cerevisiae (yeast), Arabidopsis thaliana, a variety of invertebrates, mammals and Homo sapiens.

Another model that is useful for studying the interaction of melatonin and the UPS is the regulation of deiodinases, Dio2, the enzyme which converts thyroxine to the more active thyroid hormone, triiodothyronine (T3), and Dio3, the enzyme which deiodinates T4 and T3 to inactive metabolites. The role of ubiquitination and deubiquitination in activation and deactivation of Dio2 is well documented [20, 228]. Dio2 is ubiquitinated by the ubiquitin ligases WSB-1 and TEB4 (depending on the tissue) [59] and deubiquitinated by USP20 and USP33 [19]. Their control of Dio2 has been described as an on/off switch which regulates T3 levels in various organs and tissues [55].

Melatonin injections reduced Dio2 expression [202, 274] in the hypothalamus of the Syrian hamster. The mechanisms have not been determined. In mice, daily melatonin injections reduced Dio2 expression but induced Dio3 expression [71]. In the Siberian hamster a single injection of melatonin towards the end of the light phase of the photoperiodic cycle was reported to induce Dio3 expression in the hypothalamus and its expression increased in proportion to the number of successive days of melatonin injection. Changes in photoperiod can also very rapidly change the expression of Dio2 and Dio3 in hamsters [83].

In summary, there is strong evidence that melatonin administration has an impact on the UPS. While the mechanism of this interaction is not clear, it does appear to influence the regulation of many different proteins, particularly those with a short half-life. The models discussed herein provide some information on the mechanism by which melatonin interacts with the UPS. The common features of those models are that they provide strong clues as to the mechanisms by which melatonin interacts 
with the UPS. Recently melatonin has been associated with epigenetics. A ubiquitin ligase involved in epigenetic modification is HDAC4 [285] and there is some evidence that melatonin influences protein levels of HDAC4 (see the section below).

\section{Epigenetics}

Epigenetic regulation is manifested as a heritable change in gene expression or activity without any alteration in the DNA sequence. Epigenetic modifications can be stably transmitted from a parent cell to the daughter cell (termed mitotic inheritance) or between generations (termed meiotic inheritance). Epigenetic changes have been described in pregnancy and embryonic development, cancer, neurological disorders, cardiotoxicity and hypertension, diabetes, aging and cellular senescence.

Molecular mechanisms underlying epigenetic regulation include posttranslational modification of nuclear histones by enzymes such as histone acetyl transferases (HAT) or histone deacetylases (HDAC) or methylation of cytosine bases in DNA by a family of DNA methyl transferases (DNMT) [123]. Non-coding RNAs (ncRNAs), including lncRNA and miRNA, can also modulate gene expression and are considered to be epigenetic modifiers (Fig. 5). All these molecular mechanisms allow additional control over gene expression.

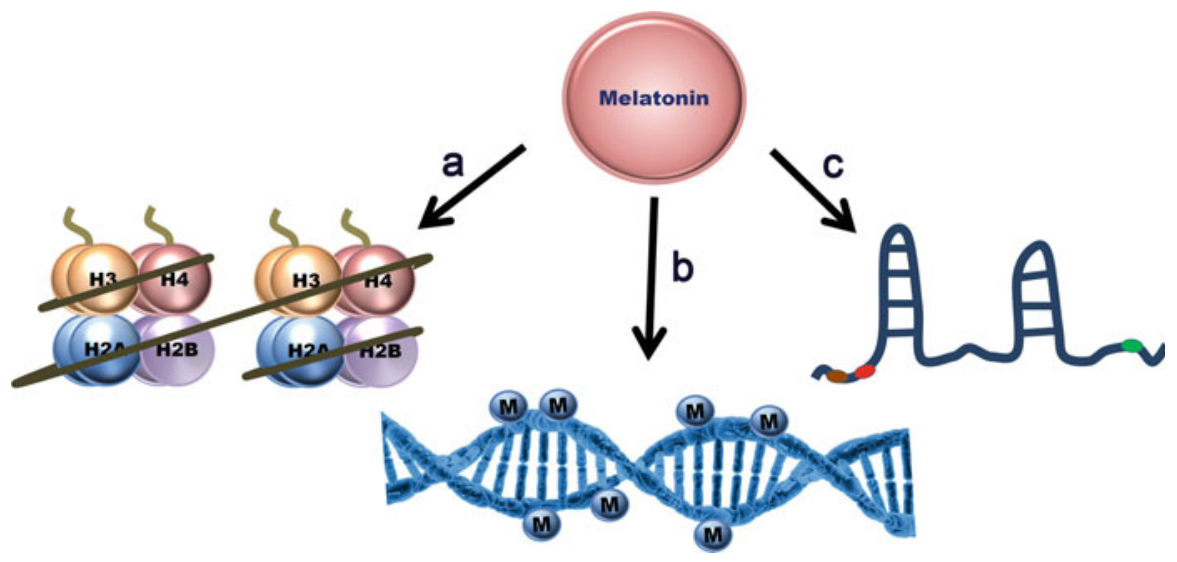

Fig. 5 Molecular mechanisms underlying epigenetic regulation. (a) An octameric core of histones (dimers of H2A, H2B, H3 and H4) forms a nucleosome. When DNA is not being transcribed, it is tightly wound around the nucleosomes. Acetylation of lysine residues in the histones, catalyzed by HATs, allow accessibility to RNA polymerase II. After transcription, the histones are deacetylated and returned to a closed chromatin state. (b) Addition of methyl groups to carbon-5 position of cytosine residues in $\mathrm{CpG}$ islands within the promoter or coding regions of genes alters their binding affinity to transcription factors. Hypermethylation generally leads to gene silencing whereas hypomethylation generally leads to gene activation (c) Non-coding RNAs (ncRNAs), including lncRNA and miRNA, can also modulate gene expression and are considered to be epigenetic modifiers 
Histones are basic proteins located within the nucleus; an octameric core of histones (dimers of $\mathrm{H} 2 \mathrm{~A}, \mathrm{H} 2 \mathrm{~B}, \mathrm{H} 3$ and $\mathrm{H} 4$ ) forms a nucleosome. When DNA is not being transcribed, it is tightly wound around the nucleosomes, preventing the binding and initiation of transcription by RNA polymerase II. For transcription, this "closed" chromatin structure has to be "opened" for it to be accessible; this occurs by acetylation of lysine residues in the histones, catalyzed by HATs. Acetylated lysine residues are recognized by protein complexes containing bromodomains that modulate chromatin architecture. When transcription is complete, acetylated lysine residues of histone are deacetylated by HDACs, returning chromatin to a closed state. In addition to acetylation-deacetylation, reversible methylation by histone methyltransferases and histone demethylases, reversible phosphorylation by kinases and phosphatases, mono- ubiquitination, sumoylation of lysine residues that inhibits acetylation, glycosylation and ADP ribosylation are also known to occur.

DNA methylation of carbon-5 position of cytosine residues in the cytosineguanosine (CG)-rich sequences (referred to as "CpG islands") within the promoter or coding regions of genes alters their binding affinity to transcription factors, typically resulting in repression of gene expression. DNA methyltransferase 1 (DNMT1) is the major enzyme involved in transmitting methylation patterns during replication in adults whereas DNMT3a and DNMT3b play critical roles during early development. DNMT3L does not have methyltransferase activity; however, it helps DNMT3a and DNMT3b in propagation and establishment of maternally imprinted genes. Hypermethylation generally leads to gene silencing whereas hypomethylation generally leads to gene activation. While DNA methylation is considered to be relatively permanent, histone modification is more environmentally responsive.

The enzymes involved in the biosynthesis of melatonin as well as its target membrane receptors are modulated epigenetically [78]. In turn, melatonin can purportedly inhibit DNMTs by inhibiting their transcription or by impeding their function via binding to their catalytically active sites. Melatonin also appears to induce gene expression by acetylation of histone H3; conversely, perhaps as a negative feedback loop, it promotes the expression of HDAC3, HDAC5 and HDAC7, leading to gene repression [217]. The most widely reported mechanism that links melatonin to epigenetic regulation is via its induction of sirtuins (silent information regulators), a family of seven known class III NAD + -dependent HDACS (SIRT1 to SIRT7). In normal cells, melatonin is known to upregulate SIRT1 expression and/or mitochondrial SIRT3 under various conditions $[23,76]$. Melatonin-mediated induction of SIRT1 expression contributes to protection from oxidative stress in ischemic models, age-related senescence, hypertension and inflammation [78, 161, 162].

The epigenetic role of melatonin in providing protection or biological adaptation to environmental factors that can be passed from one generation to the next via oocytes or sperms is well-documented $[105,106]$. Melatonin appears to be particularly suitable in serving as an environmental sensor [106], given that its secretion is affected by different wavelengths of light [192], temperature [160], altitude [116] and seasonal cycles [85]. Recent literature indicates that melatonin protects 
spermatogonial stem cells from endocrine disruptors such as bisphenol A or diethylhexyl phthalate by maintaining histone $\mathrm{H} 3 \mathrm{~K} 9$ dimethylation and promotes their recovery [284]. Similarly, melatonin also protects spermatogonial stem cells in mice from hexavalent chromium, another environmental carcinogen, by preventing trimethylation of histone $\mathrm{H} 3 \mathrm{~K} 9$ or $\mathrm{H} 3 \mathrm{~K} 27$, thereby preventing germ cell apoptosis and maintaining fertility [148, 287]. Protective epigenetic mechanisms underlying antioxidant defense of germ cells was demonstrated by a recent study showing hypomethylation of SOD1, Gpx4 and Cat genes in ovine prepubertal cumulus cells of lambs treated with melatonin that resulted in decreased apoptosis [64]. Surprisingly, melatonin treatment also upregulated $D N M T 1, D N M T 3 a$ and $D N M T 3 b$ in ovine prepubertal cumulus cells, with the latter gene being significantly hypomethylated [63], suggesting selective hypomethylation of the antioxidant genes. DNMTIa expression is upregulated in melatonin-treated oocytes along with increased expression of oocyte maturation- related genes, GDF9 and MARF, suggesting a beneficial epigenetic role for melatonin in oocyte maturation [251]. Melatonin also appears to reduce apoptosis of bovine somatic cell nuclear transfer embryos by stimulating SOD1 and Gpx4 expression that was associated with higher H3K9ac levels [230].

Melatonin functions by binding to one of two membrane-bound G-protein seven transmembrane receptors, MTNR1A (or MT1) and MTNR1B (or MT2) [225], or to nuclear orphan receptors from the retinoid orphan receptor (ROR) or the retinoid $\mathrm{Z}$ receptor (RZR) families [60]. Melatonin may also interact with cytosolic proteins that in turn may regulate the nuclear receptors or the cytoskeleton [26]. Interestingly, one of the SNPs linked to night shift-related job exhaustion is associated with changes in DNA methylation in the $5^{\prime}$ regulatory region of MTNR1A that may lead to decreased melatonin signaling [232].

Epigenetic regulation is known to play a role in asthma and allergy that have TH2 immune cell activation in common [148]. A recent study that performed a genomewide linkage scan of 615 European families to assess co-occurrence of asthma and allergy identified a "differentially methylated $\mathrm{CpG}$ site located within intron 1 of the melatonin receptor 1A (MTNR1A) gene that mediated the effect of a paternally transmitted genetic variant. Melatonin also appears to play a role in alleviating chronic obstructive pulmonary disease (COPD) by upregulating the expression of SIRT1 that, in turn, inhibits interleukin-1B and NLRP3-mediated inflammation [183]. Melatonin-induced SIRT1 induction also plays an anti-inflammatory role during lipopolysaccharide-induced oxidative stress [216]. Melatonin-mediated epigenetic suppression of pro-inflammatory NF-kB is reviewed elsewhere $[125,126]$.

The association between chronodisruption with consequent lower levels of melatonin and the incidence of cancer has long been noted. Recent data support a melatonin-mediated upregulation in global methylation as a key factor in tumor suppression [75, 214]. In contrast with its function in normal cells, melatonin appears to downregulate SIRT1 in cancer cells and thereby decreases their proliferative capacity [112]. Importantly, treatment of a breast cancer cell line, MCF-7, with 
melatonin followed by mapping of the epigenome identified several thousand differentially methylated genes [133], indicating an epigenetic tumor-suppressing role for melatonin in cancer. In breast cancer, the tumor suppressor gene, brcal, the DNA repair gene, rad9, and the cell cycle regulation genes, $d k k 3$ and wifl, are hyper methylated, leading to their silencing; hypomethylation of oncogenes and transposable elements such as Alu are associated with poor prognosis. Chronodisruption in shift workers leads to clock hypomethylation and cry 2 hypermethylation, as also found in breast cancer [289]. However, direct epigenetic links between chronodisruption and breast cancer are yet to be firmly established [124]. Interestingly, Mtnrla mRNA expression is reduced in patients with oral squamous cell carcinoma; its re-expression in tumor cells inhibited growth in vitro, suggesting a tumor suppressor role for melatonin in oral cancer [168]. Moreover, melatonin suppresses senescent cancer cell-mediated secretion of pro-inflammatory factors by inhibiting PARP-1interaction with the long non-coding RNA, TERRA, thereby preventing H2BK120 acetylation [276]. Finally, melatonin suppresses microRNA, miR-24, post- trancriptionally and thereby inhibits cell proliferation and migration [166].

Melatonin also regulates chromatin remodeling in the nervous system. For example, treatment with melatonin in drinking water induced acetylation of histones $\mathrm{H} 3$ and $\mathrm{H} 4$ primarily in the hippocampus that correlated with increased levels of phospho-ERK [170]. It can enhance hippocampal neurogenesis via agonists of MT1 and MT2 that appear to function via stimulation of BDNF [226]. Exogenous melatonin also enhances neurogenesis in mice during aging [190]. In addition to neurogenesis, melatonin has anti-aging neural effects that is also linked to its upregulation of SIRT1 [74]. Importantly, it protects the hippocampus from pre-natal glucocorticoid exposure by downregulating the expression of DNA methyltransferase 1 mRNA expression and by suppressing DNMT1 and methylCpG binding protein 2 ( $\mathrm{MeCP} 2$ ) binding to the reln promoter, thereby restoring the decreased levels of reln and GAD1 mRNA expression [146]. Melatonin also ameliorates neuropathic allodynia by promoting HDAC4 dephosphorylation and nuclear import with consequent upregulation of hmgbl transcription [141]. Finally, valproate, used to treat epilepsy and bipolar disorder, promotes histone $\mathrm{H} 3$ acetylation of the MT1 promoter that results in its upregulated expression [22].

Programmed hypertension that can occur due to stressful conditions pre-birth lead to epigenetic alterations in the kidney that were reversed by feeding melatonin to pregnant dams; melatonin upregulated HDAC-2, HDAC-3 and HDAC-8 in the kidneys of offspring from calorie-restricted dams [239, 240] and altered the expression of approximately 450 genes. The study also showed that melatonin upregulated Dnmt3A, Hdac4, Hdac7, Hdacll, Chd1, Chd2, Chd3, Brpf3, Bazlb and Wdrl during nephrogenesis that are all involved in epigenetic regulation [239].

Taken together, these examples suggest a role for melatonin in modulating the expression of several genes through epigenetic regulation. The findings have implications when melatonin is used as a treatment for human diseases. 


\section{Collagen and Extracellular Matrix}

Fibrosis, the pathological accumulation of collagen and extracellular matrix (ECM), interferes with the physiology of organs. Damaging fibrosis is most commonly reported in the lung [203], heart [169], liver [104], and kidney [172] but it occurs in other situations where inflammation is rampant [94] as well as in the form of adhesions [14] among organs after surgical intervention, infection, oxidative stress, etc. Whereas there is some evidence that fibrosis may be reversible, particularly in the early stages, advanced fibrosis is associated with end stage disease, organ failure and death. To overcome the latter two events, the only available treatment is organ transplantation. Initially, fibrosis is a physiological reparative process but, when it continues to expand, it becomes pathological and life threatening (Fig. 6). Thus, limiting the sustained development of pro-fibrotic processes is medically critical.

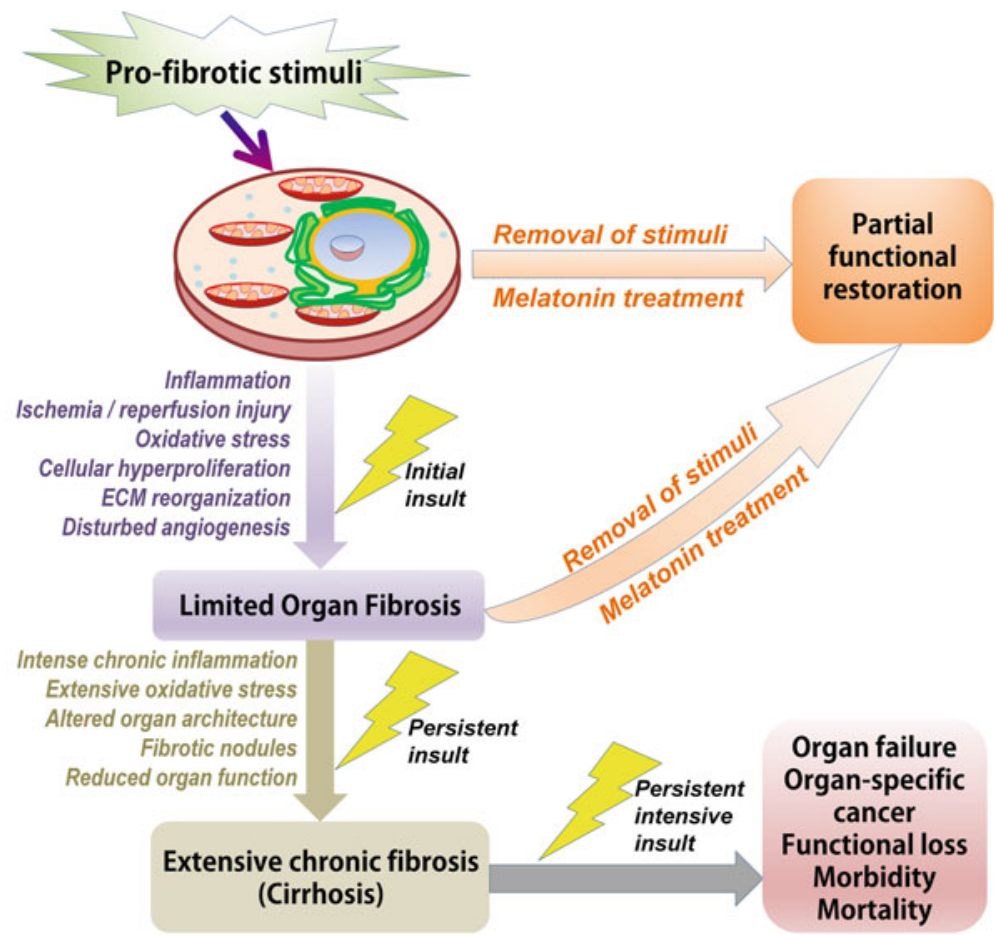

Fig. 6 Profibrotic stimuli, which are numerous, if sustained only for a brief interval cause minor organ fibrosis. Some evidence suggests that, at this point, if the fibrotic stimulus is interrupted or if melatonin treatment is initiated, the developing fibrogenic phenotype may be reversed with partial morphological and functional restoration of the organ. With more persistent attack and excessive fibroproliferative conditions, the "point-of-no-return" is exceeded and extensive fibrosis and cirrhosis occur. Eventually, organ failure is the result leading to total organ function failure and death if the organ is not replaced 
The sequence of events for the fibrotic changes that occur in different organs are similar at the molecular level with common pathological pathways that culminate in serious negative outcomes [97]. Fibrosis is initiated by a variety of pro-fibrotic processes; some of the most common are inflammation (e.g., macrophages and $\mathrm{T}$ cells) and oxidative stress. The latter process may be the result of a different events such as ischemia/reperfusion injury, toxin exposure, ionizing radiation damage, etc. Dysfunctional epithelial cells and inflammatory cells, following their recruitment to the site of injury, become fibrosis-initiator cells, which transform and actively proliferate. These activated cells include fibroblasts/myofibroblasts and other collagen-generating elements that are derived from organ-specific cells that undergo epithelial-to-mesenchymal transition. In some organs there are other cells that contribute collagen and extracellular matrix deposition, e.g., stellate cells of the liver [238].

Investigations into the role of melatonin in resisting fibrosis have uncovered several means by which this agent impedes excessive collagen and extracellular matrix (ECM) deposition. Of special interest is that melatonin membrane receptors (MT1 and MT2) are widely expressed on fibroblasts in developing scar tissue. In contrast, fibroblasts derived from skin dermal tissue lack melatonin receptors. These findings suggest that, at least in part and under some conditions, melatonin probably controls the activity of hyperfunctional fibroblasts/myofibroblasts via receptormediated mechanisms [97]. Given the high damaging reactive oxygen species generation by inflammatory cells and cells crippled by ironizing radiation, drugs, etc., melatonin's receptor-independent actions may also be involved in suppressing the development of fibrosis $[66,80]$. For example, melatonin limits the epithelial-tomesenchymal transition of lung cells exposed to bleomycin [286] and during leptinmediated fibrosis in the heart [157].

Melatonin is acknowledged as a potent anti-inflammatory agent. The recruitment of inflammatory cells to the site of the fibrotic cascade is due to locally produced cytokines. Pro- inflammatory cytokines that aid in mediating the inflammatory cascade include numerous agents such as interleukins (IL), IL-1, IL-6, IL-20 and others. Also, tumor necrosis factor-alpha (TNF- $\alpha$ ) actively participates in attracting inflammatory cells [237].

Transforming growth factor-beta (TGF- $\beta$ ) is referred to as the "master regulator of fibrosis" [164]. TGF- $\beta 1$ is a major common driving force for fibrosis in many organs and its actions involve multiple cell types. In many fibrotic disease models, blockage of TGF- $\beta 1$ reduces fibroblast activation, collagen production and ECM deposition. TGF- $\beta 1$ promotes fibrosis via both canonical and non-canonical signaling routes. Smad transcription factors are involved in the canonical pathway; these actions are highly complex because of their interactions with other signaling pathways and their ability to modulate the EMT.

In addition to TGF- $\beta$, other molecular markers of fibrosis have been identified. Smad has already been mentioned in this context. Additionally, however, PDGF (platelet-derived growth factor), CTGF (connective tissue growth factor) as well as pro-collagen-1 have been implicated to participate in pro-fibrogenic responses $[46,131]$. These fibrotic indices, as well as TGF- $\beta 1$, are influenced by a wide variety 
of interacting pathways; collectively, they drive the highly complex fibrogenic processes which lead to exaggerated collagen and ECM accumulation [159, 204].

Melatonin, in other diseases, impairs the EMT [231] and is presumed to do likewise during fibrotic events although this has been sparingly investigated. During the initiation of fibrosis, the activation of pro-fibrotic cells contribute to the accumulation of especially collagen I and glycosaminoglycans (GAG) in the extracellular space. In the early stages of the fibrotic pathway, some of the structural and functional damage can be reversed if the stimulus is withdrawn (Fig. 6) or if melatonin is used as a treatment [33, 46, 47]. Eventually, however, the severity of chronic fibrosis is so vast that the "point-of-no-return" is exceeded and organ function is essentially totally compromised thereby requiring organ transplantation. Moreover, extensive irreversible fibrosis is a prelude to other diseases, e.g., cancer [97].

The ability of melatonin to restrain fibrogenic growth has been investigated in several major organs where excessive collagen formation often occurs and where it severely compromises organ physiology and threatens the quality of life. In the case of myocardial protection, melatonin reduced scar formation in the heart after induced ischemia/reperfusion injury [57], after isoproterenol-mediated damage [157] and in other experimental conditions [97]. The respiratory system is a frequent site of fibrotic diseases including acute respiratory distress syndrome (ARDS) and COPD (chronic obstructive pulmonary disease). The damaging culprit in these conditions is often cigarette smoke. Experimentally, the regular administration of melatonin to animals exposed daily to cigarette smoke (see below) or to bleomycin [21] curtailed the severity of alveolar destruction as indicted by the amounts of oxidatively damaged protein and lipid in the lungs. In a preliminary blinded, placebo-controlled study in children with cystic fibrosis (CF), melatonin improved their wellbeing indicating it may have some benefit in individuals with this debilitating condition [54].

The most extensive experimental data regarding the efficacy of melatonin to interfere with fibrotic reactions have been studied using models of liver disease. Many conditions are accompanied by hepatic fibrosis and compromised function including a high-fat diet [277], excessive alcohol consumption [128], toluene inhalation [246], as well as the metabolism of a number of medications [47, 48]. The damaging effects of each of these conditions at the level of the liver have all been shown to be stymied by concurrent melatonin treatment. Similarly, melatonin attenuates caustic sclerosing cholangitis [215] and damage to the biliary tree resulting from bile duct ligation [9]. While the majority of these studies were not mechanistically based, melatonin's antioxidant actions seem to be the basis of some of the protection afforded by this molecule.

Two recently-published investigations specifically examined the mechanisms by which melatonin reduces the respiratory consequences of experimental chronic obstructive pulmonary disease (COPD), a condition in which pathological lung fibrosis plays a major role [171]. In the first of these, Shin and coworkers [220] performed both in vivo and in vitro studies to identify the molecular mechanisms by which melatonin blunts fibrosis. For the in vivo test, rats were exposed to cigarette 
smoke daily for a week and treated with lipopolysaccharide intravenously with or without daily melatonin treatment. At the conclusion of the study (on day 7), the non-melatonin treated rats had high numbers of inflammatory cells in their bronchoalveolar fluid (BALF) and an elevated expression of TGF- $\beta 1$, collagen I and Smad3 in their lung tissues. In a dose-response manner, melatonin inhibited each of the parameters. The in vitro study utilized a human mucoepithelial cell line treated with cigarette smoke condensate (SSC). This treatment caused an elevated expression of not only TGF- $\beta 1$ and collagen I, but also tumor necrosis factor-alpha (TNF- $\alpha$ ) and Smad 3 and p38 phosphorylation. As in the animals study, each of the indices related to fibrosis was suppressed when melatonin was added to the incubation medium.

Using a similar model of compromised respiratory function, but with a longer treatment period (28 days), Peng et al. [183] documented that melatonin preserved more normal alveolar architecture which was damaged by the combination of cigarette smoke and LPS. Moreover, in an evaluation of lung function, melatonin treatment improved the elasticity and dynamic compliance of the respiratory system while reducing the resistance to inspiration. At the molecular level, the indices of inflammation (IL-1 $\beta$ and the NLPR3 inflammasome) were reversed by melatonin, a process that is dependent on the promotion of SIRT1.

Extreme fibrosis, especially when it occurs in essential organs as illustrated herein reduces the quality of life and can lead to death. In the advanced stages, there is no currently- available medical treatment with the remaining option being organ replacement. Since it is not treatable, effort is currently directed to the prevention of fibrosis. In this regard, melatonin has become of interest as a molecule with the potential to retard or prevent the progression of the fibrotic cascade [97]. Some of the proposed mechanisms related to melatonin's inhibitory actions or the fibrotic cascade, based on the data evaluated in this report, are summarized in Fig. 7.

\section{Concluding Remarks}

The monumental effort spent by Lerner et al. [135] in the isolation and characterization of melatonin from bovine pineal tissue has paid highly significant dividends since the publication of that seminal report. The functional repertoire of melatonin is now known to far exceed that envisaged by those who investigated its actions during the first two decades after its discovery. Melatonin's functional "tool kit" include a variety of health-relevant actions such as circadian/circannual rhythm regulation, sleep promotion, immunostimulation, etc.

Within the last three decades, the list of systemic and subcellular functions where melatonin has been shown to be operative has continued to expand. Due to advances in technology, as with other molecules, research on melatonin has been focused on its intracellular functions. The preponderance of evidence suggests that melatonin is involved in some of the most basic molecular interactions within cells. The demonstration that melatonin is not uniquely of pineal origin but rather may be produced in 


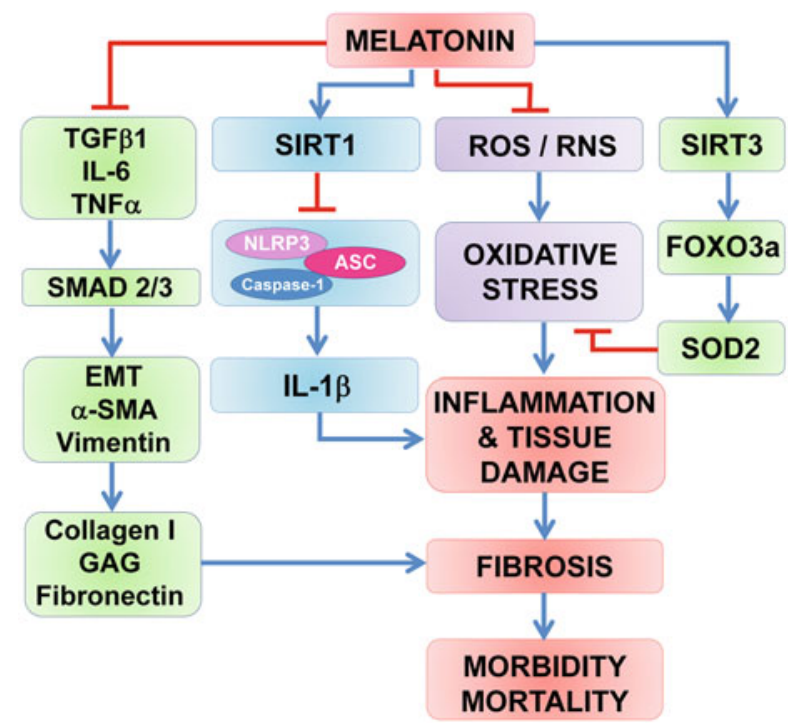

Fig. 7 Melatonin seems to interfere with fibrotic development via several means. A major factor in initiating the fibrotic cascade is transforming growth factor-1 $\beta$ (TGF-1 $\beta$ ). Melatonin inhibits TGF- $1 \beta$ and associated cytokines thereby inhibiting epithelial-to-mesenchymal transition and reducing the excessive accumulation of extracellular collagen and glycosaminoglycans (GAG). Likewise, via SIRT1 stimulation, melatonin inhibits the NLRP3 inflammasome and IL- $1 \beta$ generation. Additionally, by means of its direct radical scavenging actions and its stimulation of antioxidant enzymes, e.g., superoxide dismutase 2 (SOD2), melatonin reduces oxidative stress which normally promotes fibrosis. Many details of these pathways remain to be clarified

the mitochondria of every cell, opens vast new areas for research. It is the authors' opinion, in fact, that what is known about the intimate actions of melatonin is a small fraction of what it actually does. Moreover, past and recently discovered actions are only epiphenomenal of what melatonin does with the real actions of this molecule yet to be revealed.

In this review only a small number of the critical subcellular actions of melatonin are briefly summarized. While these functions are discussed under different headings, they are obviously interrelated and mutually dependent on each other.

The goal of subsequent research is to aggressively continue to examine the actions of this multifaceted agent; especially since many publications have strongly indicated that melatonin is a functionally flexible and highly beneficial molecule. This is apparent from the results of a number of reports where melatonin has been used at the clinical and veterinary levels. Almost uniformly, these findings show that melatonin promotes optimal function and the well-being of cells, of organs and of organisms. Finally, in plants, which also produce this molecule, melatonin's actions are equally important and life sustaining. 


\section{References}

1. Absi, E., et al. 2000. Protective effect of melatonin against the 1-methyl-4-phenylpyridiniuminduced inhibition of complex I of the mitochondrial respiratory chain. Journal of Pineal Research 29: 40-47.

2. Acuna-Castroviejo, D., et al. 1997. Melatonin is protective against MPTP-induced striatal and hippocampal lesions. Life Sciences 60: P123-P129.

3. . 2011. Melatonin-mitochondria interplay in health and disease. Current Topics in Medicinal Chemistry 11: 221-240.

4. - 2014. Extrapineal melatonin: Sources, regulation and potential functions. Cellular and Molecular Life Sciences 71: 2997-3025.

5. - 2017. Melatonin, clock genes and mitochondria in sepsis. Cellular and Molecular Life Sciences 74: 3965-3988.

6. 2018. Melatonin actions in the heart: More than a hormone. Melatonin Res 1: 21-26.

7. Ahmad, K.A., et al. 2004. Hydrogen peroxide-mediated cytosolic acidification is a signal for mitochondrial translocation of Bax during drug-induced apoptosis of tumor cells. Cancer Research 64: 7867-7878.

8. Aiken, C.T., et al. 2011. Oxidative stress-mediated regulation of proteasome complexes. Molecular \& Cellular Proteomics 10: R110 006924.

9. Aktas, C., et al. 2014. Melatonin attenuates oxidative stress, liver damage and hepatic apoptosis after bile-duct ligation in rats. Toxicology and Industrial Health 30: 835-844.

10. Alonso, M., et al. 2006. Melatonin inhibits the expression of the inducible isoform of nitric oxide synthase and nuclear factor kapa B activation in rat skeletal muscle. Journal of Pineal Research 41: 8-14.

11. Amin, A.H., et al. 2015. Melatonin ameliorates metabolic risk factors, modulates apoptotic proteins, and protects the rat heart against diabetes-induced apoptosis. European Journal of Pharmacology 747: 166-173.

12. Amir, R.E., et al. 2004. Mechanism of processing of the NF-kappa B2 p100 precursor: Identification of the specific polyubiquitin chain-anchoring lysine residue and analysis of the role of NEDD8-modification on the $\mathrm{SCF}$ (beta-TrCP) ubiquitin ligase. Oncogene 23: 2540-2547.

13. Amstrup, A.K., et al. 2013. Melatonin and the skeleton. Osteoporosis International Journal 24: 2919-2927.

14. Ara, C., et al. 2005. Protective effects of melatonin against oxidative stress on adhesion formation in the rat cecum and uterine horn model. Life Sciences 77: 1341-1350.

15. Arataki, S., et al. 2005. Calpain inhibitors prevent neuronal cell death and ameliorate motor disturbances after compression-induced spinal cord injury in rats. Journal of Neurotrauma 22: 398-406.

16. Arenzana-Seisdedos, F., et al. 1997. Nuclear localization of I kappa B alpha promotes active transport of NF-kappa B from the nucleus to the cytoplasm. Journal of Cell Science 110: 369-378.

17. Areti, A., et al. 2017. Melatonin prevents mitochondrial dysfunction and promotes neuroprotection by inducing autophagy during oxaliplatin-evoked peripheral neuropathy. Journal of Pineal Research 62: e12393.

18. Arnao, M.B., and J. Hernandez-Ruiz. 2019. Melatonin: A new plant hormone and/or a plant master regulator? Trends in Plant Science 4: 38-48.

19. Arrojo, E.D.R., et al. 2013. The type II deiodinase is retrotranslocated to the cytoplasm and proteasomes via p97/Atx3 complex. Molecular Endocrinology 27: 2105-2115.

20. - 2013. Role of the type 2 iodothyronine deiodinase (D2) in the control of thyroid hormone signaling. Biochimica et Biophysica Acta 1830: 3956-3964. 
21. Arslan, S.O., et al. 2002. The effect of melatonin on bleomycin-induced pulmonary fibrosis in rats. Journal of Pineal Research 32: 21-25.

22. Bahna, S.G., and L.P. Niles. 2017. Epigenetic regulation of melatonin receptors in neuropsychiatric disorders. British Journal of Pharmacology 175: 3209-3219.

23. Bai, X.Z., et al. 2016. Melatonin prevents acute kidney injury in severely burned rats via the activation of SIRT1. Scientific Reports 6: 32199.

24. Beinke, S., et al. 2004. Lipopolysaccharide activation of the TPL-2/MEK/extracellular signalregulated kinase mitogen-activated protein kinase cascade is regulated by lkaapaB kinaseinduced proteolysis of NF-kappaB1 p105. Molecular and Cellular Biology 24: 9658-9667.

25. Beni, S.M., et al. 2004. Melatonin0induced neuroprotection after closed head injury is associated with increased brain antioxidants and attenuated late-phase activation of NF-kappaB and AP-1. The FASEB Journal 18: 149-151.

26. Benitez-King, G., and F. Anton-Tay. 1993. Calmodulin mediates melatonin cytoskeletal effects. Experientia 49: 635-641.

27. Bizzarri, M., et al. 2013. Molecular mechanisms of the pro-apoptotic actions of melatonin in cancer: A review. Expert Opinion on Therapeutic Targets 17: 1483-1496.

28. Bragoszewski, P., et al. 2017. Control of mitochondrial biogenesis and function by the ubiquitin- proteasome system. Open Biology 7: 170007.

29. Brenner, C., et al. 2000. Bcl-2 and Bax regulate the channel activity of the mitochondrial adenine nucleotide translocator. Oncogene 19: 329-336.

30. Burchell, V.S., et al. 2013. The Parkinson's disease-linked proteins Fbxo7 and Parkin interact to mediate mitophagy. Nature Neuroscience 16: 1257-1265.

31. Burgess, H.J., and J.S. Emens. 2018. Drugs used in circadian sleep-wake rhythm disturbances. Sleep Medicine Clinics 13: 231-241.

32. Cajochen, C., et al. 2003. Role of melatonin in the regulation of human circadian rhythms and sleep. Journal of Neuroendocrinology 15: 432-437.

33. Campana, L., and J.P. Iredale. 2017. Regression of liver fibrosis. Seminars in Liver Disease 37: $1-10$.

34. Cardinali, D.P., and D.E. Vigo. 2017. Melatonin, mitochondria, and the metabolic syndrome. Cellular and Molecular Life Sciences 74: 3941-3954.

35. Champney, T.H., et al. 1984. Concurrent determination of enzymatic activities and substrate concentrations in the melatonin synthetic pathway within the same rat pineal gland. Journal of Neuroscience Research 11: 59-66.

36. Chang, C.F., et al. 2012. Melatonin attenuates kainic acid-induced neurotoxicity in mouse hippocampus via inhibition of autophagy and alpha-synuclein aggregation. Journal of Pineal Research 52: 312-321.

37. Chen, Z.X., and S. Pervaiz. 2007. Bcl-2 induces pro-oxidant state by engaging mitochondrial respiration in tumor cells. Cell Death and Differentiation 14: 1617-1627.

38. Chen, Y., et al. 2016. Melatonin induces anti-inflammatory effects to play a protective role via endoplasmic reticulum stress in acute pancreatitis. Cellular Physiology and Biochemistry 40: 1094-1104.

39. Chen, Z., et al. 2017. Effects of melatonin on maturation, histone acetylation, autophagy of porcine oocytes and subsequent embryonic development. Animal Science Journal 88: $1298-1310$.

40. Chern, C.M., et al. 2012. Melatonin ameliorates neural function by promoting endogenous neurogenesis through the MT2 melatonin receptor in ischemic-stroke mice. Free Radical Biology \& Medicine 52: 1634-1647.

41. Chrivia, J.C., et al. 1993. Phosphorylated CREB binds specifically to the nuclear protein CBP. Nature 365: 855-859.

42. Chua, S., et al. 2016. The cardioprotective effect of melatonin and exendin-4 treatment in a rat model of cardiorenal syndrome. Journal of Pineal Research 61: 438.

43. Chuang, J.I., et al. 1996. Effect of melatonin on NF-kappa-B DNA-binding activity in the rat spleen. Cell Biology International 20: 687-692. 
44. Clément, M.V., and S. Pervaiz. 1999. Reactive oxygen intermediates regulate cellular response to apoptotic stimuli: An hypothesis. Free Radical Research 30: 247-252.

45. Cory, S., and J.M. Adams. 2002. The Bcl2 family: Regulators of the cellular life-or-death switch. Nature Reviews. Cancer 2: 647-656.

46. Crespo, I., et al. 2015. Melatonin limits the expression of profibrogenic genes and ameliorates the progression of hepatic fibrosis in mice. Translational Research 165: 346-357.

47. Cruz, A., et al. 2005. Melatonin prevents experimental liver cirrhosis induced by thioacetamide in rats. Journal of Pineal Research 39: 143-150.

48. Czechowska, G., et al. 2015. Protective effects of melatonin against thioacetamide-induced liver fibrosis in rats. Journal of Physiology and Pharmacology 66: 567-579.

49. Dabbeni-Sala, F., et al. 2001. Melatonin protects against 6-OHDA-induced neurotoxicity in rats: A role for mitochondrial complex I activity. The FASEB Journal 15: 167-170.

50. - 2001. Kainic acid induces selective mitochondrial oxidative phosphorylation enzyme dysfunction in cerebellar granule neurons: Protective effects of melatonin and GSH ethyl ester. The FASEB Journal 15: 1786-1788.

51. Dardente, H., et al. 2016. Seasonal breeding in mammals: From basic research to applications and back. Theriogenology 86: 324-332.

52. de Luxan-Delgado, B., et al. 2014. Melatonin administration decreases adipogenesis in the liver of $\mathrm{Ob} / \mathrm{Ob}$ mice through autophagy modulation. Journal of Pineal Research 56: 126-133.

53. - 2016. Melatonin reduces endoplasmic reticulum stress and autophagy in liver of leptin-deficient mice. Journal of Pineal Research 61: 108-123.

54. DeCastro-Silva, C., et al. 2010. Melatonin improves sleep and reduces nitrite in the exhaled breath condensate in cystic fibrosis - A randomized double-blind placebo-controlled study. Journal of Pineal Research 48: 65-71.

55. Dentice, M., et al. 2013. The deiodinases and the control of intracellular thyroid hormone signaling during cellular differentiation. Biochimica et Biophysica Acta 1830: 3937-3945.

56. Ding, M., et al. 2018. Melatonin prevents Drp1-mediated mitochondrial fission in diabetic hearts through SIRT1-PGC1alpha pathway. Journal of Pineal Research 65: e12491.

57. Drobnik, J., et al. 2011. Pharmacological doses of melatonin reduce the glycosaminoglycan level within the infarcted heart scar. Journal of Physiology and Pharmacology 62: 29-35.

58. Dubocovich, M.L., and M. Markowska. 2005. Functional MT1 and MT2 melatonin receptors in mammals. Endocrine 27: 101-110.

59. Egri, P., and B. Gereben. 2014. Minimal requirements for ubiquitination-mediated regulation of thyroid hormone activation. Journal of Molecular Endocrinology 53: 217-226.

60. Emet, M., et al. 2016. A review of melatonin, its receptors and drugs. The Eurasian Journal of Medicine 48: 135-141.

61. Fan, J., et al. 2018. Melatonin: A multifunctional factor in plants. International Journal of Molecular Sciences 19: E1528.

62. Fan, T., et al. 2018. Inhibiting MT2-TFE3-dependent autophagy enchances melatonin-induced apoptosis in tongue squamous cell carcinoma. Journal of Pineal Research 64: 1-18.

63. Fang, Y., et al. 2018. Melatonin-mediated development of ovine cumulus cells, perhaps by regulation of DNA methylation. Molecules 23 (494): 1-14.

64. 2019. Melatonin-induced demethylation of antioxidant genes increases antioxidant capacity through Roralpha in cumulus cells of prepubertal lambs. Free Radical Biology \& Medicine 131: 173-183.

65. Fischer, T.W., et al. 2013. Melatonin enhances antioxidative enzymes gene expression (CAT, GPx, SOD), prevents their UVR-induced depletion, and protects against the formation of DNA damage (8-hydroxy-2'-deoxyguanosine) in ex vivo human skin. Journal of Pineal Research 54: $303-312$.

66. Galano, A., and R.J. Reiter. 2018. Melatonin and its metabolites vs oxidative stress: From individual actions to collective protection. Journal of Pineal Research 65: e12514.

67. Galano, A., et al. 2018. Melatonin: A versatile protector against oxidative DNA damage. Molecules 23: E530. 
68. Gastel, J.A., et al. 1998. Melatonin production: Proteasomal proteolysis in serotonin N-acetyltransferase regulation. Science 279: 1358-1360.

69. Gatfield, D., and U. Schibler. 2007. Proteasomes keep the circadian clock ticking. Science 316: $1135-1136$.

70. Gatti, G., et al. 2017. Antiproliferative and pro-apoptotic activity of melatonin analogues on melanoma and breast cancer cells. Oncotarget 8: 68338-68353.

71. Goto, M., et al. 2013. Melatonin-induced changes in the expression of thyroid hormoneconverting enzymes in hypothalamus depend on the timing of melatonin injections and genetic background in mice. General and Comparative Endocrinology 186: 33-40.

72. Gottlieb, D.J., et al. 2010. Prospective study of obstructive sleep apnea and incident coronary heart disease and heart failure: The sleep heart health study. Circulation 122: 352-360.

73. Guo, P., et al. 2014. Melatonin improves mitochondrial function by promoting MT1/SIRT1/ PGC-1 alpha-dependent mitochondrial biogenesis in cadmium-induced hepatotoxicity in vitro. Toxicological Sciences 142: 182-195.

74. Gutierrez-Cuesta, J., et al. 2008. Evaluation of potential pro-survival pathways regulated by melatonin in a murine senescence model. Journal of Pineal Research 45: 497-505.

75. Haim, A., and A.E. Zubidat. 2015. Artificial light at night: Melatonin as a mediator between the environment and epigenome. Philosophical Transactions of the Royal Society of London. Series B, Biological Sciences 370: 20140121.

76. Han, D., et al. 2016. Melatonin facilitates adipose-derived mesenchymal stem cells to repair the murine infarcted heart via the SIRT1 signaling pathway. Journal of Pineal Research 60: 178-192.

77. Hardeland, R. 2013. Melatonin and the theories of aging: A critical appraisal of melatonin's role in antiaging mechanisms. Journal of Pineal Research 55: 325-356.

78. - 2014. Melatonin, noncoding RNAs, messenger RNA stability and epigeneticsevidence, hints, gaps and perspectives. International Journal of Molecular Sciences 15: $18221-18252$

79. - 2017. Melatonin and the electron transport chain. Cellular and Molecular Life Sciences 74: 3883-3896.

80. - 2018. Recent findings in melatonin research and their relevance to the CNS. Central Nervous System Agents in Medicinal Chemistry 18: 102-104.

81. Hayden, M.S., and S. Ghosh. 2004. Signaling to NF-kappaB. Genes \& Development 18: $2195-2224$.

82. He, C., et al. 2016. Mitochondria synthesize melatonin to ameliorate its function and improve mice oocyte's quality under in vitro conditions. International Journal of Molecular Sciences 17: E939.

83. Herwig, A., et al. 2012. Hypothalamic gene expression rapidly changes in response to photoperiod in juvenile Siberian hamsters (Phodopus sungorus). Journal of Neuroendocrinology 24: 991-998.

84. Hevia, D., et al. 2015. Melatonin uptake in prostate cancer cells: Intracellular transport versus simple passive diffusion. Journal of Pineal Research 45: 247-257.

85. Hewing, M. 1984. Seasonal variations in the cerebrospinal fluid-contacting area of the pineal gland in the golden hamster (Mesocricetus auratus). Anatomy and Embryology (Berlin) 169: 91-96.

86. Hill, S.M., et al. 2015. Melatonin: An inhibitor of breast cancer. Endocrine-Related Cancer 32: 183-204.

87. Hirpara, J.L., et al. 2001. Intracellular acidification MV triggered by mitochondrial-derived hydrogen peroxide is an effector mechanism for drug-induced apoptosis in tumor cells. The Journal of Biological Chemistry 276: 514-521.

88. Histing, T., et al. 2012. Melatonin impairs fracture healing by suppressing RANKL-mediated bone remodeling. The Journal of Surgical Research 173: 83-90.

89. Ho, A.K., and C.L. Chik. 2010. Modulation of Aanat gene transcription in the rat pineal gland. Journal of Neurochemistry 112: 321-331. 
90. Ho, A.K., et al. 2006. Opposite effects of proteasome inhibitors in the adrenergic induction of arylalkylamine $\mathrm{N}$-acetyltransferase in rat pinealocytes. Chronobiology International 23: 361-367.

91. Hochrainer, K., et al. 2015. The ubiquitin ligase HERC3 attenuates NF-kappaB-dependent transcription independently of its enzymatic activity by delivering the RelA subunit for degradation. Nucleic Acids Research 43: 9889-9904.

92. Hoesel, B., and J.A. Schmid. 2013. The complexity of NF-kappaB signaling in inflammation and cancer. Molecular Cancer 12: 86.

93. Hong, Y., et al. 2014. Melatonin treatment induces interplay of apoptosis, autophagy, and senescence in human colorectal cancer cells. Journal of Pineal Research 56: 264-274.

94. Hosseinzadeh, A., et al. 2018. Idiopathic pulmonary fibrosis (IPF) signaling pathways and protective roles of melatonin. Life Sciences 201: 17-29.

95. Hsu, S.Y., et al. 1996. Targeted overexpression of Bcl-2 in ovaries of transgenic mice leads to decreased follicle apoptosis, enhanced folliculogenesis, and increased germ cell tumorigenesis. Endocrinology 137: 4837-4843.

96. Hsu, S.M., et al. 2015. Proteasome inhibitor bortezomib suppresses nuclear factor-kappa B activation and ameliorates eye inflammation in experimental autoimmune uveitis. Mediators of Inflammation 2015: 847373.

97. Hu, W., et al. 2016. Melatonin: The dawning of a treatment for fibrosis? Journal of Pineal Research 60: 121-131.

98. Hu, J., et al. 2017. Melatonin alleviates postinfarction cardiac remodeling and dysfunction by inhibiting Mst12. Journal of Pineal Research 62: e12368-e12381.

99. Huang, S.H., et al. 2008. Melatonin decreases TLR3-mediated inflammatory factor expression via inhibition of NF-kappa B activation in respiratory syncytial virus-infected RAW264.7 macrophages. Journal of Pineal Research 45: 93-100.

100. Huang, Z., et al. 2010. N-terminal residues regulate proteasomal degradation of AANAT. Journal of Pineal Research 48: 290-296.

101. Huo, X., et al. 2017. Human transporters, PEPT1/2, facilitate melatonin transportation into mitochondria of cancer cells: An implication of the therapeutic potential. Journal of Pineal Research 62: e12390.

102. Hussain, A.R., et al. 2009. Proteasome inhibitor MG-132 mediated expression of p27Kip1 via S-phase kinase protein 2 degradation induces cell cycle coupled apoptosis in primary effusion lymphoma cells. Leukemia \& Lymphoma 50: 1204-1213.

103. Indran, I.R., et al. 2011. Recent advances in apoptosis, mitochondria and drug resistance in cancer cells. Biochimica et Biophysica Acta 1807: 735-745.

104. Iredale, J.P., et al. 2013. Extracellular matrix degradation in liver fibrosis: Biochemistry and regulation. Biochimica et Biophysica Acta 1832: 876-883.

105. Irmak, M.K., and O. Ozcan. 1997. Human diversity, environmental adaptation and neural crest. Medical Hypotheses 48: 407-410.

106. Irmak, M.K., et al. 2005. Melatonin seems to be a mediator that transfers the environmental stimuli to oocytes for inheritance of adaptive changes through epigenetic inheritance system. Medical Hypotheses 64: 1138-1143.

107. Janjetovic, Z., et al. 2017. Melatonin and its metabolites protect human melanocytes against UVB-induced damage: Involvement of NRF2-mediated pathways. Scientific Reports 7: 1274.

108. Jeong, J.K., et al. 2012. Melatonin-induced autophagy protects against human prion proteinmediated neurotoxicity. Journal of Pineal Research 53: 138-146.

109. Jou, M.J., et al. 2004. Visualization of the antioxidative effects of melatonin at the mitochondrial level during oxidative stress-induced apoptosis of rat brain astrocytes. Journal of Pineal Research 37: 55-70.

110. 2007. Melatonin protects against common deletion of mitochondrial DNA- augmented mitochondrial oxidative stress and apoptosis. Journal of Pineal Research 43: 389-403. 
111. 2010. Visualization of melatonin's multiple mitochondrial levels of protection against mitochondrial $\mathrm{Ca}^{2+}$-mediated permeability transition and beyond in rat brain astrocytes. Journal of Pineal Research 48: 20-38.

112. Jung-Hynes, B., et al. 2011. Melatonin, a novel Sirt1 inhibitor, imparts antiproliferative effects against prostate cancer in vitro in culture and in vivo in TRAMP model. Journal of Pineal Research 50: 140-149.

113. Kang, J.W., et al. 2016. Melatonin enhances mitophagy and mitochondrial biogenesis in rats with carbon tetrachloride-induced liver fibrosis. Journal of Pineal Research 60: 383-393.

114. Karbowski, M., and R.J. Youle. 2011. Regulating mitochondrial outer membrane proteins by ubiquitination and proteasomal degradation. Current Opinion in Cell Biology 23: 476-482.

115. Karin, M., and Y. Ben-Neriah. 2000. Phosphorylation meets ubiquitination: The control of NF-[kappa]B activity. Annual Review of Immunology 18: 621-663.

116. Kaur, C., et al. 2002. Plasma melatonin, pinealocyte morphology, and surface receptors/ antigen expression on macrophages/microglia in the pineal gland following a high-altitude exposure. Journal of Neuroscience Research 67: 533-543.

117. Kayumov, L., et al. 2000. Melatonin, sleep and circadian rhythm disorders. Seminars in Clinical Neuropsychiatry 5: 44-55.

118. Kerr, J.F., et al. 1972. Apoptosis: A basic biological phenomenon with wide-ranging implications in tissue kinetics. British Journal of Cancer 26: 239-257.

119. Kim, J.E., et al. 2010. Suppression of NF-kappaB signaling by KEAP1 regulation of IKKbeta activity through autophagic degradation and inhibition of phosphorylation. Cellular Signalling 22: $1645-1654$.

120. Klein, D.C. 2007. Arylalkylamine N-acetyltransferase: The timezyme. The Journal of Biological Chemistry 282: 4233-4237.

121. Klein, D.C., and G.R. Berg. 1970. Pineal gland: Stimulation of melatonin production by norepinephrine involves cyclic AMP-mediated stimulation of $\mathrm{N}$-acetyltransferase. Advances in Biochemical Psychopharmacology 3: 241-263.

122. Klein, D.C., et al. 1997. The melatonin rhythm-generating enzyme: Molecular regulation of serotonin N-acetyltransferase in the pineal gland. Recent Progress in Hormone Research 52: 307-357.

123. Klose, R.J., and A.P. Bird. 2006. Genomic DNA methylation: The mark and its mediators. Trends in Biochemical Sciences 31: 89-97.

124. Kochan, D.Z., and O. Kovalchuk. 2015. Circadian disruption and breast cancer: An epigenetic link? Oncotarget 6: 16866-16882.

125. Korkmaz, A.S., et al. 2012. Gene regulation by melatonin linked to epigenetic phenomena. Gene 503: 1-11.

126. Korkmaz, G.G., et al. 2012. Melatonin ameliorates oxidative damage in hyperglycemiainduced liver injury. Clinical and Investigative Medicine 35: e370-e377.

127. Koyama, H., et al. 2002. Melatonin at pharmacologic doses increases bone mass by suppressing resorption through down-regulation of the RANKL-mediated osteoclast formation and activation. Journal of Bone and Mineral Research 17: 1219-1229.

128. Lackner, C., and D. Tiniakos. 2019. Fibrosis and alcohol-related liver disease. Journal of Hepatology 70: 294-304.

129. Lamb, C.A. 2013. The autophagosome: Origins unknown, biogenesis complex. Nature Reviews. Molecular Cell Biology 14: 759-774.

130. Lavie, J., et al. 2018. Ubiquitin-dependent degradation of mitochondrial proteins regulates energy metabolism. Cell Reports 23: 2852-2863.

131. Lawrance, I.C., et al. 2015. Cellular and molecular mediators of intestinal fibrosis. Journal of Crohn's \& Colitis 11: 1491-1503.

132. Lee, D.F., et al. 2009. KEAP1 E3 ligase-mediated downregulation of NF-kappaB signaling by targeting IKKbeta. Molecular Cell 36: 131-140. 
133. Lee, S.E., et al. 2013. Genome-wide profiling in melatonin-exposed human breast cancer cell lines identifies differentially methylated genes involved in the anticancer effect of melatonin. Journal of Pineal Research 54: 80-88.

134. Lee, S.J., et al. 2018. Melatonin inhibits apoptotic cell death induced by Vibrio vulnificus Vvha via melatonin receptor 2 coupling with NCF-1. Cell Death \& Disease 9: 48.

135. Lerner, A.B., et al. 1958. Isolation of melatonin, the pineal gland factor that lightens melanocytes. Journal of the American Chemical Society 80: 2587.

136. Li, J.H., et al. 2005. Melatonin reduces inflammatory injury through inhibiting NF-kappaB activation in rats with colitis. Mediators of Inflammation 2005: 185-193.

137. Li, H., et al. 2014. Alterations in the time course of expression of the Nox family in the brain in a rat experimental cerebral ischemia and reperfusion model: Effects of melatonin. Journal of Pineal Research 57: 110-119.

138. Li, M., et al. 2016. Cognitive dysfunction and epigenetic alterations of the BDNF gene are induced by social isolation during early adolescence. Behavioural Brain Research 313: $177-183$.

139. 2016 . Melatonin antagonizes cadmium-induced neurotoxicity by activing the transcription factor EB-dependent autophagy-lysosome machinery in mouse neuroblastoma cells. Journal of Pineal Research 61: 353-369.

140. Lignitto, L., et al. 2011. Control of PKA stability and signaling by the RING ligase praja2. Nature Cell Biology 13: 412-422.

141. Lin, T.B., et al. 2016. Melatonin relieves neuropathic allodynia through spinal Mt2-enhanced Pp2ac and downstream Hdac4 shuttling-dependent epigenetic modification of Hmgb1 transcription. Journal of Pineal Research 60: 263-276.

142. Liu, J., et al. 2016. MT1 and MT2 melatonin receptors: A therapeutic perspective. Annual Review of Pharmacology and Toxicology 56: 361-383.

143. Liu, S., et al. 2017. Evaluation of cell death pathways initiated by antitumor drugs melatonin and valproic acid in bladder cancer cells. FEBS Open Bio 7: 798-810.

144. Lowes, D.A., et al. 2013. Antioxidants that protect mitochondria reduce interleukin-6 and oxidative stress, improve mitochondrial function, and reduce biochemical markers of organ dysfunction in a rat model of acute sepsis. British Journal of Anaesthesia 110: 472-480.

145. Luchetti, F., et al. 2010. Melatonin signaling and cell protection function. The FASEB Journal 24: 3603-3624.

146. Lui, C.C., et al. 2015. Effects of melatonin on prenatal dexamethasone-induced epigenetic alterations in hippocampal morphology and reelin and glutamic acid decarboxylase 67 levels. Developmental Neuroscience 37: 105-114.

147. Luo, C., et al. 2019. The multiple protective roles and molecular mechanisms of melatonin and its precursor $\mathrm{N}$-acetylserotonin in targeting brain injury and liver damage and in maintaining bone health. Free Radical Biology \& Medicine 130: 215-233.

148. Lv, Y., et al. 2018. Melatonin protects mouse spermatogonial stem cells against hexavalent chromium-induced apoptosis and epigenetic histone modification. Toxicology and Applied Pharmacology 340: 30-38.

149. Maity, P., et al. 2009. Melatonin reduces indomethacin-induced gastric mucosal cell apoptosis by preventing mitochondrial oxidative stress and the activation of mitochondrial pathway of apoptosis. Journal of Pineal Research 46: 314-323.

150. Manchester, L.C., et al. 1995. Melatonin immunoreactivity in the photosynthetic prokaryote Rhodospirillum rubrum: Implications of an ancient antioxidant system. Cellular \& Molecular Biology Research 41: 391-395.

151. 2015. Melatonin: An ancient molecule that makes oxygen metabolically tolerable. Journal of Pineal Research 59: 403-419.

152. Maria, S., and P.A. Witt-Enderby. 2014. Melatonin effects on bone: Potential use for the prevention and treatment for osteopenia, osteoporosis and periodontal disease and for use in bone-grafting procedures. Journal of Pineal Research 56: 115-125. 
153. Martin, M., et al. 2000. Melatonin-induced increased activity of the respiratory chain complexes I and IV can prevent mitochondrial damage induced by ruthenium red in vivo. Journal of Pineal Research 28: 242-248.

154. 2000 . Melatonin but not vitamin $\mathrm{C}$ and $\mathrm{E}$ maintains glutathione homeostasis in $t$-butyl hydroperoxide-induced mitochondrial oxidative stress. The FASEB Journal 14: 1677-1679.

155. 1 2002. Melatonin increases the activity of the oxidative phosphorylation enzymes and the production of ATP in rat brain and liver mitochondria. The International Journal of Biochemistry \& Cell Biology 34: 348-357.

156. Martin, V., et al. 2014. Involvement of autophagy in melatonin-induced cytotoxicity in glioma- initiating cells. Journal of Pineal Research 57: 308-316.

157. Martinez-Martinez, E., et al. 2014. Leptin induces cardiac fibrosis through galectin-3, mTOR and oxidative stress: Potential role of obesity. Journal of Hypertension 32: 1104-1114.

158. Massa, P.T., et al. 2006. NFkappaB in neurons? The uncertainty principle in neurobiology. Journal of Neurochemistry 97: 607-618.

159. Massague, J. 2012. TGF $\beta$ signaling in context. Nature Reviews. Molecular Cell Biology 13: 616-630.

160. Matsushima, S., and Y. Morisawa. 1980. Mechanism involved in the response of granulated vesicles in the mouse pinealocyte to acute cold exposure. Cell and Tissue Research 208: 247-252.

161. Mayo, J.C., et al. 2017. Melatonin and sirtuins: A "not-so unexpected" relationship. Journal of Pineal Research 62.

162. - 2017. Melatonin transport into mitochondria. Cellular and Molecular Life Sciences 74: 3927-3940.

163. Mediavilla, M.D., et al. 2010. Basic mechanisms involved in the anti-cancer effects of melatonin. Current Medicinal Chemistry 17: 4462-4481.

164. Meng, X.M., et al. 2016. TGF- $\beta$ : The master regulator of fibrosis. Nature Reviews. Nephrology 12: 325-338.

165. Mishra, A., S. Paul, and S. Swarnakar. 2011. Downregulation of matrix metalloproteinase-9 by melatonin during prevention of alcohol-induced liver injury in mice. Biochimie 93: 854-866.

166. Mori, F., et al. 2016. Multitargeting activity of Mir-24 inhibits long-term melatonin anticancer effects. Oncotarget 7: 20532-20548.

167. Mukherjee, D., et al. 2010. Melatonin protects against isoproterenol-induced myocardial injury in the rat: Antioxidative mechanisms. Journal of Pineal Research 48: 251-262.

168. Nakamura, E., et al. 2008. Frequent silencing of a putative tumor suppressor gene melatonin receptor 1 a (MTNR1A) in oral squamous-cell carcinoma. Cancer Science 99: 1390-1400.

169. Nduhirabandi, F., and G.J. Maarman. 2018. Melatonin in heart failure: A promising therapeutic strategy? Molecules 23: 1819-1837.

170. Niles, L.P., et al. 2013. Melatonin induces histone hyperacetylation in the rat brain. Neuroscience Letters 541: 49-53.

171. Nobakht, M., et al. 2015. The metabolomics of airway diseases, including COPD, asthma and cystic fibrosis. Biomarkers 20: 5-16.

172. Nogueira, A., et al. 2017. Pathophysiological mechanisms of renal fibrosis: A review of animal models and therapeutic targets. In Vivo, vol. 31, 1-22.

173. Okatani, Y., et al. 2003. Protective effect of melatonin against mitochondrial injury induced by ischemia and reperfusion of rat liver. European Journal of Pharmacology 469: 145-152.

174. - 2003. Acutely administered melatonin restores mitochondrial physiology in old mice. The International Journal of Biochemistry \& Cell Biology 35: 367-375.

175. Ordonez, R., et al. 2015. Ceramide metabolism regulates autophagy and apoptotic cell death induced by melatonin in liver cancer cells. Journal of Pineal Research 59: 178-189.

176. Orogo, A.M., and A.B. Gustafsson. 2015. Therapeutic targeting of autophagy: Potential and concerns in treating cardiovascular disease. Circulation Research 116: 489-503. 
177. Osier, N.D., et al. 2017. Brain injury results in lower levels of melatonin receptor subtypes MT1 and MT2. Neuroscience Letters 650: 18-24.

178. Ostrowska, A.Z., et al. 2001. Assessment of the relationship between dynamic pattern of nighttime levels of melatonin and chosen biochemical markers of bone metabolism in a rat model of postmenopausal osteoporosis. Neuro Endocrinology Letters 22: 129-136.

179. Pan, P., et al. 2018. Melatonin balance the autophagy and apoptosis by regulating UCP2 in the LPS-induced cardiomyopathy. Molecules 23: 675.

180. Paradies, G., et al. 2017. Mitochondrial bioenergetics decay in aging: Beneficial effect of melatonin. Cellular and Molecular Life Sciences 74: 3897-3912.

181. Park, K.H., et al. 2011. Melatonin promotes osteoblastic differentiation through the BMP/ERK/Wnt signaling pathways. Journal of Pineal Research 51: 187-194.

182. Park, E.J., et al. 2014. Transcriptional and post-translational regulation of Bim controls apoptosis in melatonin-treated human renal cancer Caki cells. Journal of Pineal Research 56: $97-106$.

183. Peng, Z., et al. 2018. Melatonin attenuates ovary inflammation via SIRT1 dependent inhibition of NLRP3 inflammasome and IL-1 $\beta$ in rats with COPD. International Immunopharmacology 62: 23-28.

184. Pervaiz, S., et al. 1999. Superoxide anion inhibits drug-induced tumor cell death. FEBS Letters 59: 343-348.

185. Peter, M.E., et al. 1997. Advances in apoptosis research. Proceedings of the National Academy of Sciences of the United States of America 94: 12736-12737.

186. Pi, H., et al. 2015. SIRT3-SOD2-mROS-dependent autophagy in cadmium-induced hepatotoxicity and salvage by melatonin. Autophagy 11: 1037-1051.

187. Poeggeler, B., et al. 1994. Melatonin - A highly potent endogenous radical scavenger and electron donor: New aspects of the oxidation chemistry of this indole assessed in vitro. Annals of the New York Academy of Sciences 738: 419-420.

188. Proietti, S., et al. 2017. Melatonin, mitochondria and the cancer cell. Cellular and Molecular Life Sciences 74: 4015-4026.

189. Qin, W., et al. 2012. Melatonin inhibits IL1beta-induced MMP9 expression and activity in human umbilical vein endothelial cells by suppressing NF-kappaB activation. The Journal of Endocrinology 214: 145-153.

190. Ramirez-Rodriguez, G., et al. 2012. Melatonin supplementation delays the decline of adult hippocampal neurogenesis during normal aging of mice. Neuroscience Letters 530: 53-58.

191. Ramos, E., et al. 2017. Ischemic brain injury: New insights on the protective role of melatonin. Free Radical Biology \& Medicine 104: 32-53.

192. Reiter, R.J. 1992. Alterations of the circadian melatonin rhythm by the electromagnetic spectrum: A study in environmental toxicology. Regulatory Toxicology and Pharmacology 15: 226-244.

193. Reiter, R.J., and D.X. Tan. 2003. What constitutes a physiological concentration of melatonin? Journal of Pineal Research 34: 79-80.

194. Reiter, R.J., et al. 2001. Melatonin in plants. Nutrition Reviews 59: 286-290.

195. 2008. Melatonin combats molecular terrorism at the mitochondrial level. Interdisciplinary Toxicology 1: 101-113.

196. - 2014. Melatonin: Exceeding expectations. Physiology (Bethesda) 29: 325-333.

197. 2016. Melatonin as an antioxidant: Under promises but over delivers. Journal of Pineal Research 61: 253-278.

198. 2017. Melatonin as a mitochondria-targeted antioxidant: One of evolution's best ideas. Cellular and Molecular Life Sciences 74: 3863-3881.

199. - 2017. Role of SIRT3/SOD2 signaling in mediating the antioxidant action of melatonin in mitochondria. Current Trends in Endocrinology 9: 45-49.

200. 2018. Mitochondria: Central organelles for melatonin's antioxidant and anti- aging actions. Molecules 23: E509. 
201. - 2018. Historical perspective and evaluation of the mechanisms by which melatonin modulates seasonal reproduction in mammals. Melatonin Research 1: 1-17.

202. Revel, F.G., et al. 2006. Melatonin regulates type 2 deiodinase gene expression in the Syrian hamster. Endocrinology 147: 4680-4687.

203. Richeldi, L., et al. 2007. Idiopathic pulmonary fibrosis. Lancet 389: 1941-1952.

204. Rockey, D.C., et al. 2015. Fibrosis - A common pathway to organ injury and failure. The New England Journal of Medicine 372: 1138-1149.

205. Rodriguez, C., et al. 2004. Regulation of antioxidant enzymes: A significant role for melatonin. Journal of Pineal Research 36: 1-9.

206. Rosales-Corral, S.A., et al. 2012. Alzheimer's disease: Pathological mechanisms and the beneficial role of melatonin. Journal of Pineal Research 52: 167-202.

207. Russo, A., et al. 2010. Bortezomib: A new pro-apoptotic agent in cancer treatment. Current Cancer Drug Targets 10: 55-67.

208. Sack, R.L., and A.J. Lewy. 1997. Melatonin as a chronobiotic: Treatment of circadian desynchrony in night workers and the blind. Journal of Biological Rhythms 12: 595-603.

209. Samantaray, S., et al. 2008. Melatonin attenuates calpain upregulation, axonal damage and neuronal death in spinal cord injury in rats. Journal of Pineal Research 44: 348-357.

210. Sanchez-Hidalgo, M., et al. 2009. Age-related changes in melatonin synthesis in rat extrapineal tissues. Experimental Gerontology 44: 328-334.

211. San-Miquel, B., et al. 2014. Melatonin modulates the autophagic response in acute liver failure induced by the rabbit hemorrhagic disease virus. Journal of Pineal Research 56: 313-321.

212. 2015. Melatonin inhibits autophagy and endoplasmic reticulum stress in mice with carbon tetrachloride-induced fibrosis. Journal of Pineal Research 59: 151-162.

213. Schomerus, C., et al. 2000. Selective adrenergic/cyclic AMP-dependent switch-off of proteasomal proteolysis along switches on neural signal transduction: An example from the pineal gland. Journal of Neurochemistry 75: 2123-2132.

214. Schwimmer, H., et al. 2014. Light at night and melatonin have opposite effects on breast cancer tumors in mice assessed by growth rates and global DNA methylation. Chronobiology International 31: 144-150.

215. Sezer, A., et al. 2010. Effects of intraperitoneal melatonin on caustic sclerosing cholangitis due to scolicidal solution in a rat model. Current Therapeutic Research, Clinical and Experimental 71: 118-128.

216. Shah, S.A., et al. 2017. Melatonin stimulates the SIRT1/Nrf2 signaling pathway counteracting lipopolysaccharide (LPS)-induced oxidative stress to rescue postnatal rat brain. CNS Neuroscience \& Therapeutics 23: 33-44.

217. Sharma, R., et al. 2008. Epigenetic targets for melatonin: Induction of histone H3 hyperacetylation and gene expression in C17.2 neural stem cells. Journal of Pineal Research 45: 277-284.

218. Sheen, J.M., et al. 2016. Melatonin alleviates liver apoptosis in bile duct ligation young rats. International Journal of Molecular Sciences 17: e1365.

219. Shi, D., et al. 2012. Melatonin suppresses proinflammatory mediators in lipopolysaccharidestimulated CRL1999 cells via targeting MAPK, NF-kappaB, c/EBPbeta, and p300 signaling. Journal of Pineal Research 53: 154-165.

220. Shin, N.R., et al. 2017. Melatonin suppresses fibrotic responses induced by cigarette smoke via downregulation of TGF- $\beta 1$. Oncotarget 8: 95692-95703.

221. Shiu, S.Y., et al. 2013. Melatonin MT1 receptor-induced transcriptional up-regulation of $\mathrm{p} 27$ (Kip1) in prostate cancer antiproliferation is mediated via inhibition of constitutively active nuclear factor kappa B (NF-kappaB): Potential implications on prostate cancer chemoprevention and therapy. Journal of Pineal Research 54: 69-79.

222. Shringarpure, R., et al. 2003. Ubiquitin conjugation is not required for the degradation of oxidized proteins by proteasome. The Journal of Biological Chemistry 278: 311-318.

223. Simonneaux, V., et al. 2006. Rat and Syrian hamster: Two models for the regulation of AANAT gene expression. Chronobiology International 23: 351-359. 
224. Sishi, B.J., et al. 2013. Autophagy upregulation promotes survival and attenuates doxorubicininduced cardiotoxicity. Biochemical Pharmacology 85: 124-134.

225. Slominski, R.M., et al. 2012. Melatonin membrane receptors in peripheral tissues: Distribution and functions. Molecular and Cellular Endocrinology 351: 152-166.

226. Soumier, A., et al. 2009. Mechanisms contributing to the phase-dependent regulation of neurogenesis by the novel antidepressant, agomelatine, in the adult rat hippocampus. Neuropsychopharmacology 34: 2390-2403.

227. Stehle, J.H., et al. 2011. A survey of molecular details in the human pineal gland in the light of phylogeny, structure, function and chronobiological diseases. Journal of Pineal Research 51: 17-43.

228. Steinsapir, J., et al. 1998. Type 2 iodothyronine deiodinase in rat pituitary tumor cells is inactivated in proteasomes. Journal of Clinical Investigation 102: 1895-1899.

229. Stojkovic, K., et al. 2014. A central role for ubiquitination within a circadian clock protein modification code. Frontiers in Molecular Neuroscience 7: 1-7.

230. Su, J., et al. 2015. Melatonin significantly improves the developmental competence of bovine somatic cell nuclear transfer embryos. Journal of Pineal Research 59: 455-468.

231. Su, S.C., et al. 2017. Cancer metastasis: Mechanisms of inhibition by melatonin. Journal of Pineal Research 62: e12370.

232. Sulkava, S., et al. 2017. Common genetic variation near melatonin receptor $1 \mathrm{~A}$ gene linked to job-related exhaustion in shift workers. Sleep 40: 1-10.

233. Suofu, Y., et al. 2017. Dual role of mitochondria in producing melatonin and driving GPCR signaling to block cytochrome c release. Proceedings of the National Academy of Sciences of the United States of America 114: E7997-E8006.

234. Suwanjang, W., et al. 2010. The protective effect of melatonin on methamphetamine-induced calpain-dependent death pathway in human neuroblastoma SH-SY5Y cultured cells. Journal of Pineal Research 48: 94-101.

235. . 2012. Calpastatin reduces calpain and caspase activation in methamphetamineinduced toxicity in human neuroblastoma SH-SY5Y cultured cells. Neuroscience Letters 526: 49-53.

236. 2013. Melatonin attenuates dexamethasone toxicity-induced oxidative stress, calpain and caspase activation in human neuroblastoma SH-SY5Y cells. The Journal of Steroid Biochemistry and Molecular Biology 138: 116-122.

237. Sziksz, E., et al. 2015. Fibrosis related inflammation mediators: Role of the IL-10 cytokine family. Mediators of Inflammation 2015: 764641.

238. Tahan, G., et al. 2010. Melatonin ameliorates liver fibrosis induced by bile-duct ligation in rats. Canadian Journal of Surgery 53: 313-318.

239. Tain, Y.L., et al. 2014. Transcriptional regulation of programmed hypertension by melatonin: An epigenetic perspective. International Journal of Molecular Sciences 15: 18484-18495.

240. 2 2014. Melatonin therapy prevents programmed hypertension and nitric oxide deficiency in offspring exposed to maternal caloric restriction. Oxidative Medicine and Cellular Longevity 2014: 283180.

241. Tamtaji, O.R., et al. 2019. Melatonin, a calpain inhibitor in the central nervous system: Current status and future perspectives. Journal of Cellular Physiology 234: 1001-1007.

242. Tan, D.X., and R.J. Reiter. 2019. Mitochondria: The birth place, the battle ground and the site of melatonin metabolism in cells. Melatonin Res 2: 44-66.

243. Tan, D.X., et al. 2003. Mechanistic and comparative studies of melatonin and classic antioxidants in terms of their interactions with the ABTS cation radical. Journal of Pineal Research 34: $249-259$.

244. - 2013. Mitochondrial and chloroplasts as the original sites of melatonin synthesis: A hypothesis related to melatonin's primary function and evolution in eukaryotes. Journal of Pineal Research 54: 127-138. 
245. Tan, J., et al. 2014. Melatonin protects the esophageal epithelial barrier by suppressing the transcription, expression and activity of myosin light chain kinase through ERK1/2 signal transduction. Cellular Physiology and Biochemistry 34: 2117-2127.

246. Tas, U., et al. 2011. Hepatotoxic activity of toluene inhalation and protective role of melatonin. Toxicology and Industrial Health 27: 465-473.

247. Teng, Y.C., et al. 2015. Melatonin ameliorates arsenite-induced neurotoxicity: Involvement of autophagy and mitochondria. Molecular Neurobiology 52: 1015-1022.

248. Terriff, D.L., et al. 2005. Proteasomal proteolysis in the adrenergic induction of arylalkylamine- N-acetyltransferase in rat pinealocytes. Endocrinology 146: 4795-4803.

249. Thiyagarajan, M., et al. 2008. Activated protein C promotes neovascularization and neurogenesis in post ischemic brain via protease-activated receptor 1. The Journal of Neuroscience 28: 12788-12797.

250. Thu, K.L., et al. 2011. Genetic disruption of KEAP1/CUL3 E3 ubiquitin ligase complex components is a key mechanism of NF-kappaB pathway activation in lung cancer. Journal of Thoracic Oncology 6: 1521-1529.

251. Tian, H., et al. 2012. Keap1: One stone kills three birds Nrf2, IKKbeta and Bcl-2/Bcl-xl. Cancer Letters 325: 26-34.

252. Trivedi, P.P., et al. 2016. Melatonin modulated autophagy and Nrf2 signaling pathways in mice with colitis-associated colon carcinogenesis. Molecular Carcinogenesis 55: 255-267.

253. Uygur, R., et al. 2016. Protective effects of melatonin against arsenic-induced apoptosis and oxidative stress in rat testes. Toxicology and Industrial Health 32: 848-859.

254. Vakifahmetoglu-Norberg, H., and B. Zhivotovsky. 2010. The unpredictable caspase-2: What can it do? Trends in Cell Biology 20: 150-159.

255. Vega-Naredo, I., et al. 2012. Melatonin modulates autophagy through a redox-mediated action in female Syrian hamster Harderian gland controlling cell types and gland activity. Journal of Pineal Research 52: 80-92.

256. Venegas, C., et al. 2011. Extrapineal melatonin: Analysis of its subcellular distribution and daily fluctuations. Journal of Pineal Research 52: 217-227.

257. Villela, D., et al. 2014. Norepinephrine activates NF-kappaB transcription factor in cultured rat pineal gland. Life Sciences 94: 122-129.

258. Vriend, J., and R.J. Reiter. 2014. Melatonin and ubiquitin: What's the connection? Cellular and Molecular Life Sciences 71: 3409-3418.

259. - 2014. Melatonin as a proteasome inhibitor. Is there any clinical evidence? Life Sciences 115: 8-14.

260. - 2015. The Keap1-Nrf2-antioxidant response element pathway: A review of its regulation by melatonin and the proteasome. Molecular and Cellular Endocrinology 401: 213-220.

261. - 2015. Melatonin feedback on clock genes: A theory involving the proteasome. Journal of Pineal Research 58: 1-11.

262. - 2016. Melatonin and the von Hippel-Lindau/HIF-1 oxygen sensing mechanism: A review. Biochimica et Biophysica Acta 1865: 176-183.

263. Vriend, J., et al. 2017. The pineal gland: A model for adrenergic modulation of ubiquitin ligases. PLoS One 12: e0172441.

264. Wang, Y., and S. Zeng. 2018. Melatonin promotes ubiquitination of phosphorylated proapoptotic protein Bcl-2-interacting mediator of cell death-extra long (BimEL) in porcine granulosa cells. International Journal of Molecular Sciences 19: 3431-3446.

265. Wang, S.J., et al. 2012. Melatonin suppresses apoptosis and stimulates progesterone production by bovine granulosa cells via its receptors (MT1 and MT2). Theriogenology 78: $1517-1526$.

266. Wang, F., et al. 2015. Cytoprotective effect of melatonin against hypoxia/serum deprivationinduced cell death of bone marrow mesenchymal stem cells in vitro. European Journal of Pharmacology 748: 157-165. 
267. Wang, D., et al. 2016. Bortezomib sensitizes esophageal squamous cancer cells to radiotherapy by suppressing the expression of HIF-1alpha and apoptosis proteins. Journal of X-Ray Science and Technology 24: 639-646.

268. Wongprayoon, P., and P. Govitrapong. 2017. Melatonin as a mitochondrial protector against neurodegenerative diseases. Cellular and Molecular Life Sciences 74: 3999-4014.

269. Xie, S., et al. 2015. Melatonin protects against chronic intermittent hypoxia-induced cardiac hypertrophy by modulating autophagy though the $5^{\prime}$ adenosine monophosphate-activated protein kinase pathway. Biochemical and Biophysical Research Communications 464: 975-981.

270. Xiong, J. 2015. Atg7 in development and disease: Panacea or Pandora's box? Protein \& Cell 6: 722-734.

271. Yalcin, A., et al. 2003. Apoptosis in cerebellar granule neurons is associated with reduced interaction between CREB-binding protein and NF-kappaB. Journal of Neurochemistry 84: 397-408.

272. Yamamoto, M., et al. 2018. The KEAP1-NRF2 system: A thiol-based sensor-effector apparatus for maintaining redox homeostasis. Physiological Reviews 98: 1169-1203.

273. Yaron, A., et al. 1998. Idenfication of the receptor component of the lkappaBalpha-ubiquitin ligase. Nature 396: 590-594.

274. Yasuo, S., et al. 2007. Temporal dynamics of type 2 deiodinase expression after melatonin injections in Syrian hamsters. Endocrinology 148: 4385-4392.

275. Yerlikaya, A. 2012. Expression of heme oxygenase-1 in response to proteasomal inhibition. Protein and Peptide Letters 19: 1330-1333.

276. Yu, S., et al. 2017. Melatonin regulates PARP1 to control the senescence-associated secretory phenotype (SASP) in human fetal lung fibroblast cells. Journal of Pineal Research 63: 1-18.

277. Zaitone, S., et al. 2011. Pentoxifylline and melatonin in combination with pioglitazone ameliorate experimental non-alcoholic fatty liver disease. European Journal of Pharmacology 662: 70-77.

278. Zavodnik, I.B., et al. 2011. Melatonin and succinate reduce rat liver mitochondrial dysfunction in diabetes. Journal of Physiology and Pharmacology 62: 641-647.

279. Zawilska, J.B. 1996. Melatonin as a chemical indicator of environmental light-dark cycle. Acta Neurobiologiae Experimentalis 56: 757-767.

280. Zhang, Y., et al. 2013. Melatonin inhibits the caspase-1/cytochrome c/caspase-3 cell death pathway, inhibits mt1 receptor loss and delays disease progression in a mouse model of amyotrophic lateral sclerosis. Neurobiology of Disease 55: 26-35.

281. Zhang, M., et al. 2017. Melatonin protects against diabetic cardiomyopathy through Mst1/ Sirt3 signaling. Journal of Pineal Research 63: e12418.

282. Zhang, Q., et al. 2017. Years of NF-kappaB: A blossoming of relevance to human pathobiology. Cell 168: 37-57.

283. Zhang, S., et al. 2017. Melatonin as a promising agent of regulating stem cell biology and its application in disease therapy. Pharmacological Research 117: 252-260.

284. Zhang, T., et al. 2018. Melatonin protects prepuberal testis from deleterious effects of bisphenol A or diethylhexyl phthalate by preserving H3K9 methulation. Journal of Pineal Research 65: e12497.

285. Zhao, X., et al. 2005. Regulation of MEF2 by histone deacetylase 4- and SIRT1 deacetylasemediated lysine modifications. Molecular and Cellular Biology 25: 8456-8464.

286. Zhao, H., et al. 2014. Melatonin inhibits endoplasmic reticulum stress and epithelial- mesenchymal transition during bleomycin-induced pulmonary fibrosis in mice. PLoS One 9: e97266.

287. Zheng, W., et al. 2018. In utero exposure to hexavalent chromium disrupts rat fetal testis development. Toxicology Letters 299: 201-209.

288. Zhou, H., et al. 2012. Melatonin protects against rotenone-induced cell injury via inhibition of Omi and Bax-mediated autophagy in Hela cells. Journal of Pineal Research 52: 120-127.

289. Zhu, Y., et al. 2011. Epigenetic impact of long-term shiftwork: Pilot evidence from circadian genes and whole-genome methylation analysis. Chronobiology International 28: 852-861. 